\title{
APP promotes osteoblast survival and bone formation by regulating mitochondrial function and preventing oxidative stress
}

\author{
Jin-Xiu Pan 1,2,3,4 Fulei Tang ${ }^{2,3}$, Fei Xiong ${ }^{5}$, Lei Xiong ${ }^{1,2,3,4}$, Peng Zeng ${ }^{1,2}$, Bo Wang ${ }^{1,2}$, Kai Zhao ${ }^{1,2}$, Haohan Guo ${ }^{1,2,3}$, \\ Cui Shun ${ }^{6}$, Wen-Fang Xia ${ }^{6}$, Lin Mei ${ }^{1,2,3,4}$ and Wen-Cheng Xiong ${ }^{1,2,3,4}$
}

\begin{abstract}
Amyloid precursor protein (APP) is ubiquitously expressed in various types of cells including bone cells. Mutations in App gene result in early-onset Alzheimer's disease (AD). However, little is known about its physiological function in bone homeostasis. Here, we provide evidence for APP's role in promoting bone formation. Mice that knocked out App gene $\left(\mathrm{APP}^{-1-}\right.$ ) exhibit osteoporotic-like deficit, including reduced trabecular and cortical bone mass. Such a deficit is likely due in large to a decrease in osteoblast (OB)-mediated bone formation, as little change in bone resorption was detected in the mutant mice. Further mechanical studies of APP $^{-1-}$ OBs showed an impairment in mitochondrial function, accompanied with increased reactive oxygen species (ROS) and apoptosis. Intriguingly, these deficits, resemble to those in Tg2576 animal model of AD that expresses Swedish mutant APP (APPswe), were diminished by treatment with an anti-oxidant NAC (n-acetyl-I-cysteine), uncovering ROS as a critical underlying mechanism. Taken together, these results identify an unrecognized physiological function of APP in promoting OB survival and bone formation, implicate APPswe acting as a dominant negative factor, and reveal a potential clinical value of NAC in treatment of AD-associated osteoporotic deficits.
\end{abstract}

\section{Introduction}

Alzheimer's disease (AD) is the most common form of dementia, affecting $10 \%$ of people over 65 years old ${ }^{1}$. Osteoporosis is characterized by low bone mineral density (BMD) and micro-architectural deterioration of bone tissue. $\mathrm{AD}$ and osteoporosis are largely seen as independent disorders; however, a positive association of $\mathrm{AD}$ with reduced radiographic $\mathrm{BMD}^{2,3}$ and an increase in bone resorption ${ }^{4}$ have been reported. Multiple risk genes/loci identified in $\mathrm{AD}$ patients encode proteins critical for bone

\footnotetext{
Correspondence: W.-C. Xiong (Wen-Cheng.Xiong@case.edu)

${ }^{1}$ Department of Neuroscience, Case Western Reserve University, Cleveland, $\mathrm{OH}$ 44106, USA

${ }^{2}$ Department of Neuroscience and Regenerative Medicine, Medical College of Georgia, Augusta University, Augusta, GA 30912, USA

Full list of author information is available at the end of the article.

Edited by P. Pinton
}

homeostasis, such as ApoEs4, TREM2, CD33, PYK2, VPS35, and SorL $1^{5-7}$. A degree of comorbidity of both AD and osteoporosis is also supported by epidemiological studies. Both are multifactorial and polygenetic diseases, involving aging, environmental factors, chronic inflammation, and oxidative stress as their pathogenic mechanisms. However, it remains open if and how AD is linked with osteoporosis.

App, a mendelian gene for early-onset AD (EOAD), encodes a transmembrane protein, which can be cleaved by three proteases $(\alpha, \beta \text {, and } \gamma \text {-secretases })^{8-10}$. Mutations in App gene identified in EOAD patients (e.g., Swedish mutation, APPswe) promote $A \beta$ generation ${ }^{11-13}$. Is APPswe a risk factor for AD-associated bone loss or osteoporosis? In our previous publications ${ }^{14,15}$, we have employed two transgenic mouse models, Tg2576 and

\section{(c) The Author(s) 2018}

(c) (i) Open Access This article is licensed under a Creative Commons Attribution 4.0 International License, which permits use, sharing, adaptation, distribution and reproduction in any medium or format, as long as you give appropriate credit to the original author(s) and the source, provide a link to the Creative Commons license, and indicate if changes were made. The images or other third party material in this article are included in the article's Creative Commons license, unless indicated otherwise in a credit line to the material. If material is not included in the article's Creative Commons license and your intended use is not permitted by statutory regulation or exceeds the permitted use, you will need to obtain permission directly from the copyright holder. To view a copy of this license, visit http://creativecommons.org/licenses/by/4.0/. 
a

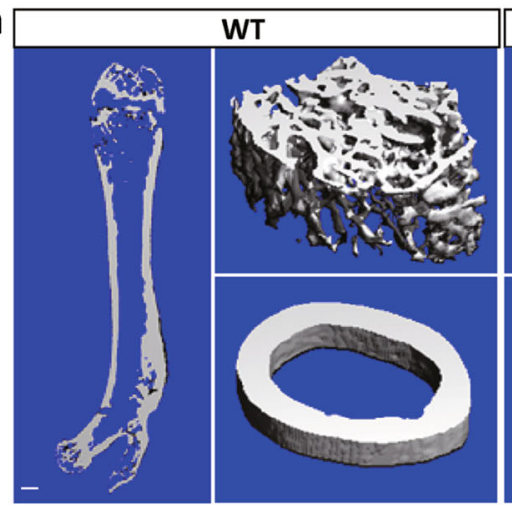

c

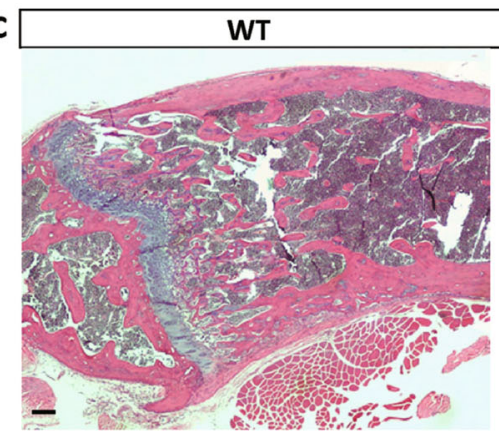

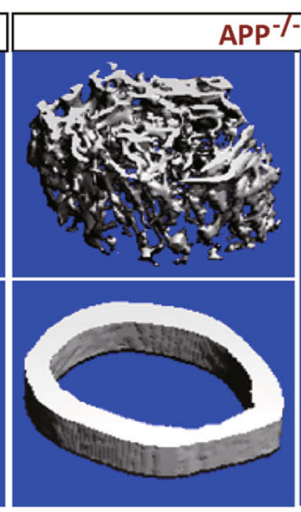
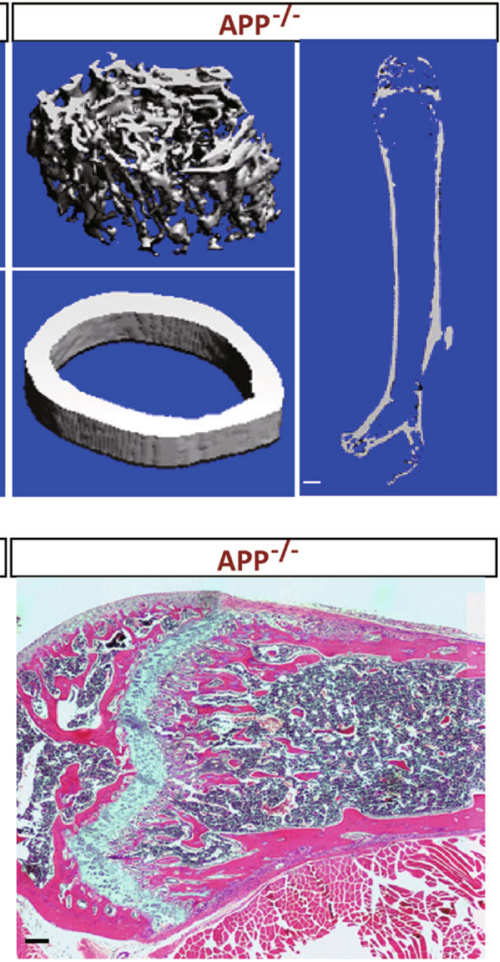
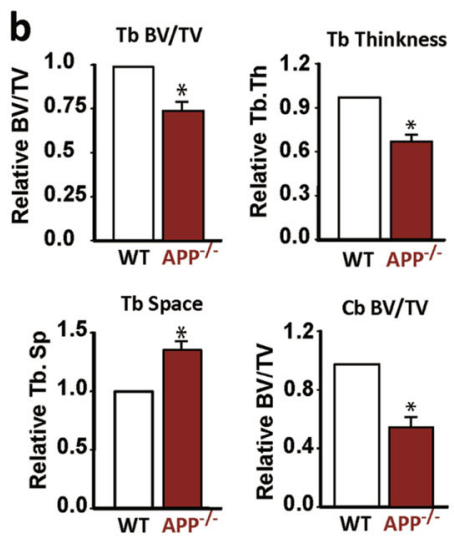

d
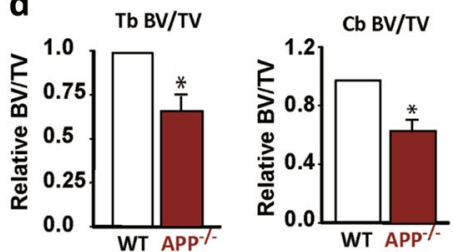

Fig. 1 Loss of trabecular and cortical bone mass in APP ${ }^{-1-}$ femurs. Five different mice (2-month old, males) per genotype per assay were examined blindly. Female mice were also characterized, and similar phenotypes as those of male mutant mice were detected, but these data were not included here. $\mathbf{a}, \mathbf{b}$ The $\mu \mathrm{CT}$ analysis of femurs from 2-M-old APP ${ }^{-1-}$ and WT mice. Representative 3-D images were shown in (a), scale bar, 1 mm. Quantification analyses (mean \pm SEM, $n=5$ ) were presented in $\mathbf{b}$. ${ }^{*} p<0.05$. Note that the trabecular bone (Tb) and cortical bone $(\mathrm{Cb})$ volumes over total volumes (BV/TV), trabecular thickness (Tb, Th) and trabecular separation (Tb.Sp) were all deficient in APP ${ }^{-/-}$femurs as compared with WT controls. c, d H \& E staining analysis of femurs from 2-M old WT and APP ${ }^{-1-}$ mice. Representative images were shown in c. Scale bar, 100 um. Quantification analyses (mean \pm SEM, $n=5$ ) were presented in $\mathbf{d}$. ${ }^{*} p<0.05$

TgAPPswe-Ocn, to address this question. Tg2576 expresses APPswe ubiquitously under the control of prion promotor ${ }^{14}$, and TgAPPswe-Ocn selectively expresses APPswe in OB-lineage cells, which is under the control of osteocalcin (Ocn) promotor driven-Cre ${ }^{15}$. Both mouse models show osteoporotic deficits and impaired $\mathrm{OB}$ differentiation and function, suggesting a cell autonomous and deleterious effect of APPswe on OB differentiation, and raising a question about APP's physiological role in this event.

Here, we determined APP's physiological function in bone homeostasis by use of APP knock out $\left(\mathrm{APP}^{-1-}\right)$ mice $^{16}$. Similar osteoporotic deficits as those of $\mathrm{Tg} 2576$ and TgAPPswe-Ocn mice were detected. Further cellular mechanical studies suggest a critical role of APP in promoting $\mathrm{OB}$ mitochondrial function, and preventing cytochrome $\mathrm{C}$ release, reactive oxygen species (ROS) production, and $\mathrm{OB}$ cell death. Intriguingly, as that in Tg2576 mice, the deficits in $\mathrm{APP}^{-1-}$ mice were diminished by treatment with NAC (n-acetyl-L-cysteine), an anti-oxidant, implicating ROS as a denominator for skeleton deficits in both Tg2576 and $\mathrm{APP}^{-/-}$mice.
These results uncover a physiological role of APP in promoting $\mathrm{OB}$ survival, bone formation, and bone homeostasis, implicate a dominant negative role of APPswe in this event, and affirm NAC's potential clinical value in the treatment of $\mathrm{AD}$-associated osteoporotic disorders.

\section{Results \\ Osteoporotic deficits in $\mathrm{APP}^{-1-}$ mice}

$\mathrm{APP}^{-1-}$ mice appeared to be smaller in body size, with a reduced body weight (Fig. S1a-b). APP was detected not only in mouse brain, but also in bone marrow cells (BMSCs and BMMs), which was abolished in cells from $\mathrm{APP}^{-1-}$ mice (Fig. S1c). These results confirmed APP's expression in bone cells as previous reports ${ }^{14,15}$, and verified $\mathrm{APP}^{-1-}$ mouse identity.

We then compared the lone bone (femur) mass between WT and $\mathrm{APP}^{-1-}$ mice by microCT $(\mu \mathrm{CT})$ analysis. Reductions in both trabecular and cortical bone volumes over total volumes were detected in $\mathrm{APP}^{-1-}$ mice, as compared with that of WT controls (Fig. 1a, b). Further analysis revealed decreased trabecular thickness (Tb. Th.) 
and increased trabecular space (Tb. Sp.) in $\mathrm{APP}^{-1-}$ mice (Fig. 1b). The reduced trabecular and cortical bone volumes in $\mathrm{APP}^{-1-}$ mice were further confirmed by $\mathrm{H}$ \& E histological analysis (Fig. 1c, d and data not shown). These results demonstrate an osteoporotic deficit in $\mathrm{APP}^{-1-}$ mice, which resemble to that of Tg2576 mice ${ }^{14,15}$, revealing a role of APP in maintaining bone homeostasis.

\section{Increase of bone resorption in neonatal, but not adult, APP $^{-1-}$ mice}

To understand how APP regulates bone homeostasis, we first examined OC-mediated bone resorption, a critical event for bone homeostasis ${ }^{17}$, in 1-M and 2-M old WT and $\mathrm{APP}^{-1-}$ mice. Measuring serum levels of PYD (pyridinoline) (a marker for bone resorption) ${ }^{18}$ showed little difference, if there is any, between WT and $\mathrm{APP}^{-1-}$ mice at age of 2-M or older (Fig. S2a and data not shown). But, at age of 1-M old, an elevated levels of serum PYD was detected in $\mathrm{APP}^{-1-}$ mice (Fig. S2a). These results suggest a transient increase of bone resorption in the neonatal age of $\mathrm{APP}^{-1-}$ mice. Further examining $\mathrm{TRAP}^{+} \mathrm{OCs}$ in $1-\mathrm{M}$ and 2-M old mice also showed a transient increase in TRAP $^{+}$ OCs per unit bone surface area in 1-M, but not 2-M, old $\mathrm{APP}^{-1-}$ mice (Fig. S2b-c). Moreover, $\mathrm{TRAP}^{+}$multi-nuclei cells (MNCs), which were in vitro differentiated from BMMs of WT and $\mathrm{APP}^{-1-}$ mice (1-M old), were increased in $\mathrm{APP}^{-1-}$ cultures in response to RANKL treatment for 3-7 days, but not 7 days after RANKL treatment (Fig. S2d, e). These results suggest that APP may play an inhibitory role in $\mathrm{OC}$-genesis and function transiently and/ or age dependently, which may contribute in part to the osteoporotic deficit in young $\mathrm{APP}^{-/-}$mice.

\section{Reduction of bone formation in $\mathrm{APP}^{-1-}$ mice}

Next, we examined OB-mediated bone formation by the following assays. First, measuring serum levels of osteocalcin (a maker for bone formation) with ELISA assay showed a reduction in $\mathrm{APP}^{-1-}$ mice (at various ages, including 1-M, 2-M, and 5-M old) (Fig. 2a and data not shown), suggesting an age-independent decrease in bone formation. Second, Goldner's trichrome staining analysis displayed a decrease in osteoid numbers per unit bone surface in $\mathrm{APP}^{-1-}$ femurs (Fig. 2b, c), in line with the view for APP to promote OB function. Third, dynamic measurements of non-decalcified femur and tibia sections, which were double-labeled by two injections of fluorescent calcein green and alizarin red S separately (at 10day interval), showed significant reductions in MAR (mineral apposition rate) and BFR (bone formation rate) in endocortical (Ec.) bone and trabecular bone (TB) in $\mathrm{APP}^{-1-}$ mice (2-M old), as compared with that of same aged WT controls (Fig. 2d-f), providing additional evidence for APP's function in bone formation. Finally, we compared OB-mediated bone formation between control and $\mathrm{APP}^{-1-}$ mice by use of the Ocn-Cre;Ai9 (Ocn;Td) reporter mice, in which, tdTomato's expression depends on osteocalcin $(\mathrm{Ocn})$ promoter driven Cre. In agreement, the tdTomato ${ }^{+} \mathrm{OBs}$ in both trabecular and cortical bone regions were marked reduced in $\mathrm{APP}^{-1-}$ mice, compared with controls (Ocn-Cre;Ai9) (Fig. 2g-j). Collectively, these results demonstrate a positive role of APP in OBmediated bone formation, which may contribute in large to the osteoporotic deficit in young as well as in aged $\mathrm{APP}^{-1-}$ mice.

\section{Decreases of OB-differentiation and function in $\mathrm{APP}^{-/-}$-BMSC cultures}

To determine if APP regulates OB-mediated bone formation in a cell autonomous manner, in vitro OB differentiation assay by culturing BMSCs derived from 2-M-old $\mathrm{WT}$ and $\mathrm{APP}^{-1-}$ mice were carried out. Indeed, OB-differentiations, viewed by ALP (alkaline phosphatase) enzymatic activity staining, were lower in both D7-OB and D14-OB cultures from BMSCs of $\mathrm{APP}^{-1-}$ mice than those of WT controls (Fig. 3a, d). Also decreased was calcified bone matrix stained by Alizarin Red $\mathrm{S}$ in $\mathrm{APP}^{-1-}-\mathrm{OB}$ cultures (Fig. 3e-f). These results suggest a cell autonomous function of APP in promoting OB-differentiation and function.

A reduced OB-differentiation is frequently associated with an increased adipogenesis ${ }^{15,19}$. Examining adipocyte differentiation (viewed by Oil Red O staining) in WT and $\mathrm{APP}^{-1-}$ BMSC cultures, however, showed no increase, but decrease, in $\mathrm{APP}^{-1-}$ cultures (Fig. 3g, h). These results implicate APP's functions in both OBs and adipocytes, rather than regulating early stage of BMSC-cell fate differentiation.

\section{Increases of apoptosis, ROS, and mitochondrial cytochrome $C$ release in $\mathrm{APP}^{-/-}-\mathrm{BM}-\mathrm{OB}$ cultures}

$\mathrm{OB}$ migration and proliferation are important events during osteogenesis, but $\mathrm{APP}^{-1-}$ has no/little effect on these events (Fig. S3). Notice that a more server reduction in OB-differentiation (viewed by ALP staining) was detected in D14- than that of D7-APP ${ }^{-1-}-\mathrm{OB}$ cultures (Fig. 3a-d); and a more server $O B$ functional deficit (viewed by Alizarin Red S) than that of OBdifferentiation defect was also observed in $\mathrm{APP}^{-1-}$ cultures (Fig. 3a-f). These observations implicate APP's function at the late stage during the OB-differentiation assay. We thus wondered if APP is critical for OBsurvival. Cell death assay by immunostaining analysis with antibodies against active caspase 3, a marker for apoptosis, was carried out. Indeed, cleaved (c)-caspase $3^{+}$cells were much more in $\mathrm{APP}^{-1-} \mathrm{OB}$ cultures than that of controls by both immunostaining and Western blot analyses (Fig. 4a-f), indicating an increase of $\mathrm{OB}$ apoptosis. Notice the c-caspase 3 was not increased in 


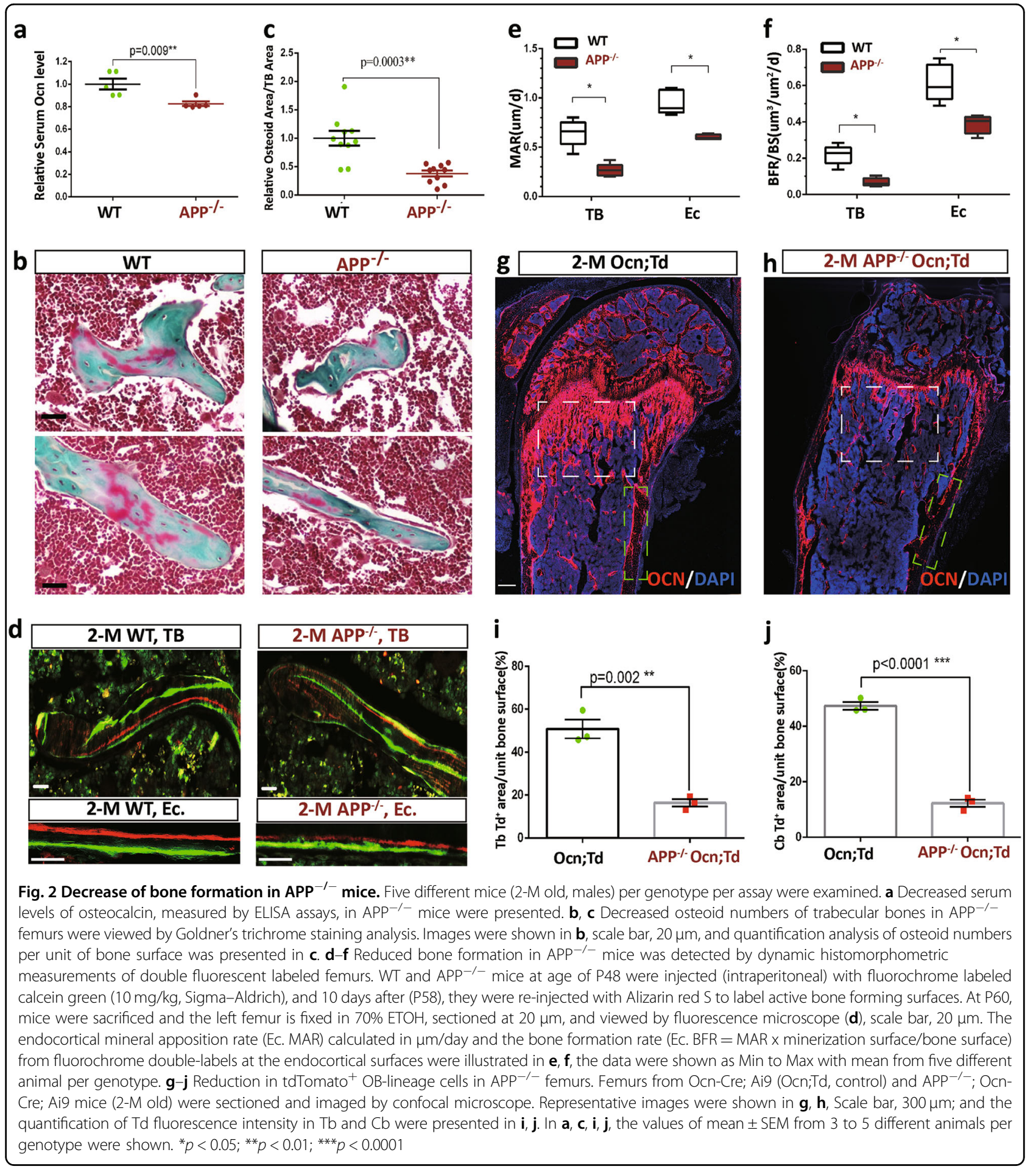

$\mathrm{APP}^{-1-}$ BMSCs (Fig. 4a-f), demonstrating a selective effect of APP. This view was further supported by the observations that the cleaved (c)-PARP1 (another marker of apoptosis) and ROS (reactive oxidative species), a cell death inducer viewed by both DCFDA fluorescence and MitoSOX Red, were all increased in $\mathrm{APP}^{-1-} \mathrm{OBs}$, but not BMSCs (Fig. 4e-l).
To understand how APP regulates OB apoptosis, we examined the canonical apoptotic pathway-activation of caspase 3 by mitochondrial released cytochrome $\mathrm{C}^{20}$. Mitochondrial and cytosol fractions were purified from lysates of BMSCs and OBs. APP was largely distributed in mitochondria ${ }^{21}$ (Fig. 5a and Fig. S4). Western blot analysis showed an increase of cytochrome $\mathrm{C}$ in cytosol fractions 

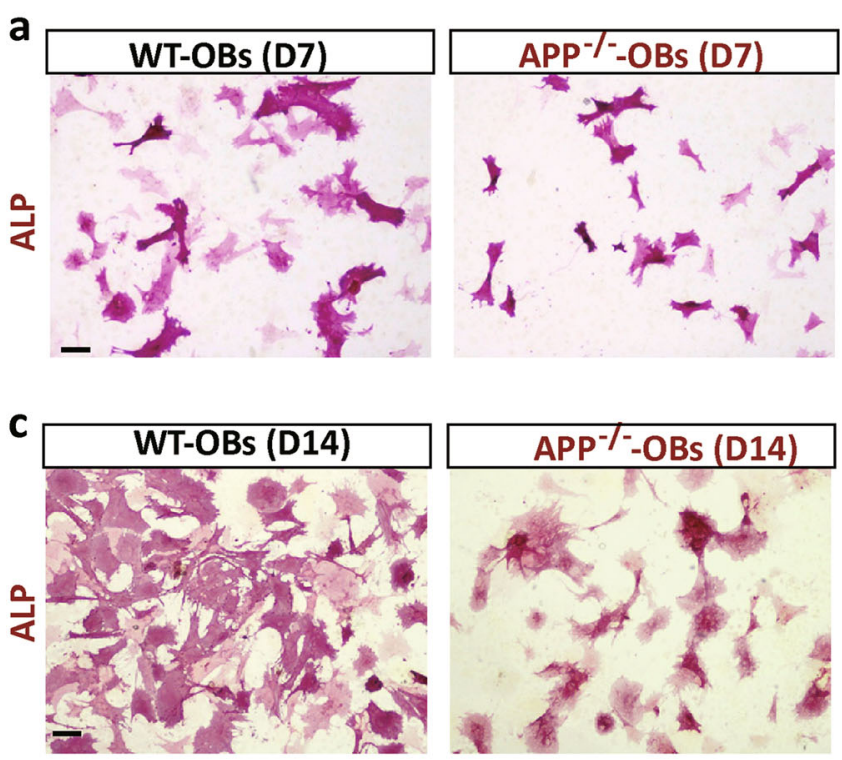

e
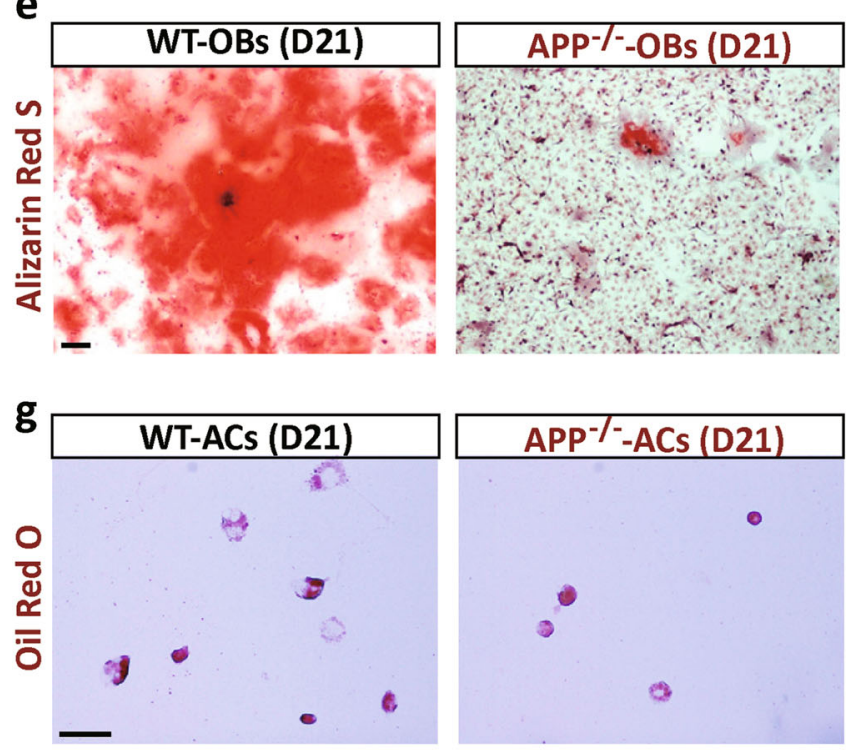
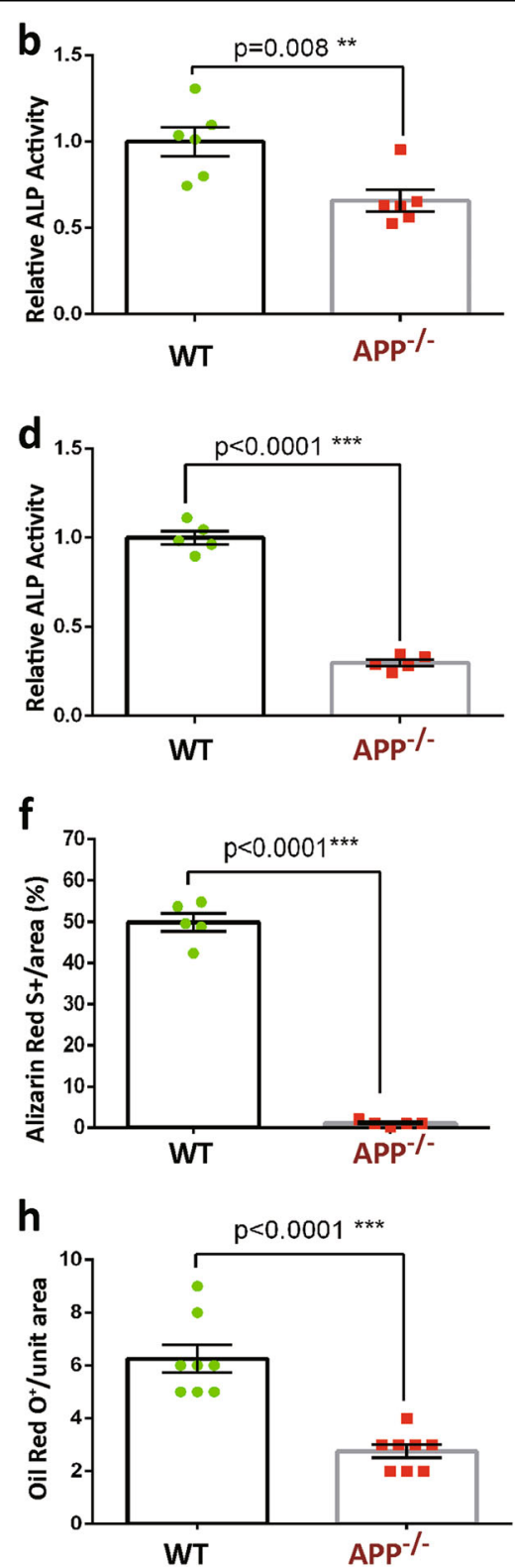

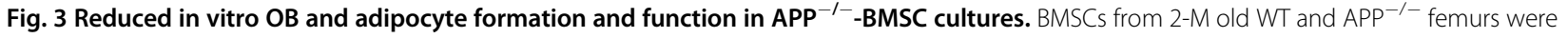
induced for $O B$ and adipocyte differentiation (see Methods). ALP staining images at day (d) 7 and 14 cultures were shown in a, $\mathbf{c}$, and ALP activities (ALP positive area/over total area) were presented in $\mathbf{b}$, $\mathbf{d}$. At D-21 culture, OB cells were stained for Alizarin Red S (e), and the Alizarin Red S staining data was quantified and illustrated in $\mathbf{f}$; and adipocytes were stained for Oil Red $\mathrm{O}(\mathbf{g})$, and Oil Red $\mathrm{O}$ staining data was quantified and presented in $\mathbf{h}$. Scale bar, $20 \mu \mathrm{m}$. In $\mathbf{b}, \mathbf{d}, \mathbf{f}$ and $\mathbf{h}$, the values of mean \pm SEM $(n=5)$ were presented. ${ }^{* *} p<0.01 ;{ }^{* * *} p<0.0001$

of OBs, but not BMSCs, from APP ${ }^{-1-}$ mice, as compared with those of WT controls (Fig. 5a-b). The cleaved caspase 3 was also elevated in the cytosol fractions of $\mathrm{APP}^{-1-}$ OBs (Fig. 5a, c). The increased cytosolic cytochrome $\mathrm{C}$ in $\mathrm{APP}^{-1-} \mathrm{OBs}$, but not BMSCs, was further examined by co-immunostaining analysis using antibodies against cytochrome $\mathrm{C}$ and Tom 20 (a mitochondrial outer membrane protein). As shown in Fig. $5 \mathrm{e}-\mathrm{h}$, the mitochondrial cytochrome $\mathrm{C}$ was lower, but the mitochondrial un- associated cytochrome $\mathrm{C}$ was higher, in $\mathrm{APP}^{-1-} \mathrm{OBs}$, compared with that of WT controls. Together, these results demonstrate an increase of cytochrome $\mathrm{C}$ release from mitochondria of $\mathrm{APP}^{-1-} \mathrm{OBs}$, and revealing an underlying mechanism of APP preventing OBs from cell death.

In addition to the increased mitochondrial cytochrome $\mathrm{C}$ release, the mitochondrial morphology (labeled by Tom20) appeared to be shorter and rounder in $\mathrm{APP}^{-/-}$ 


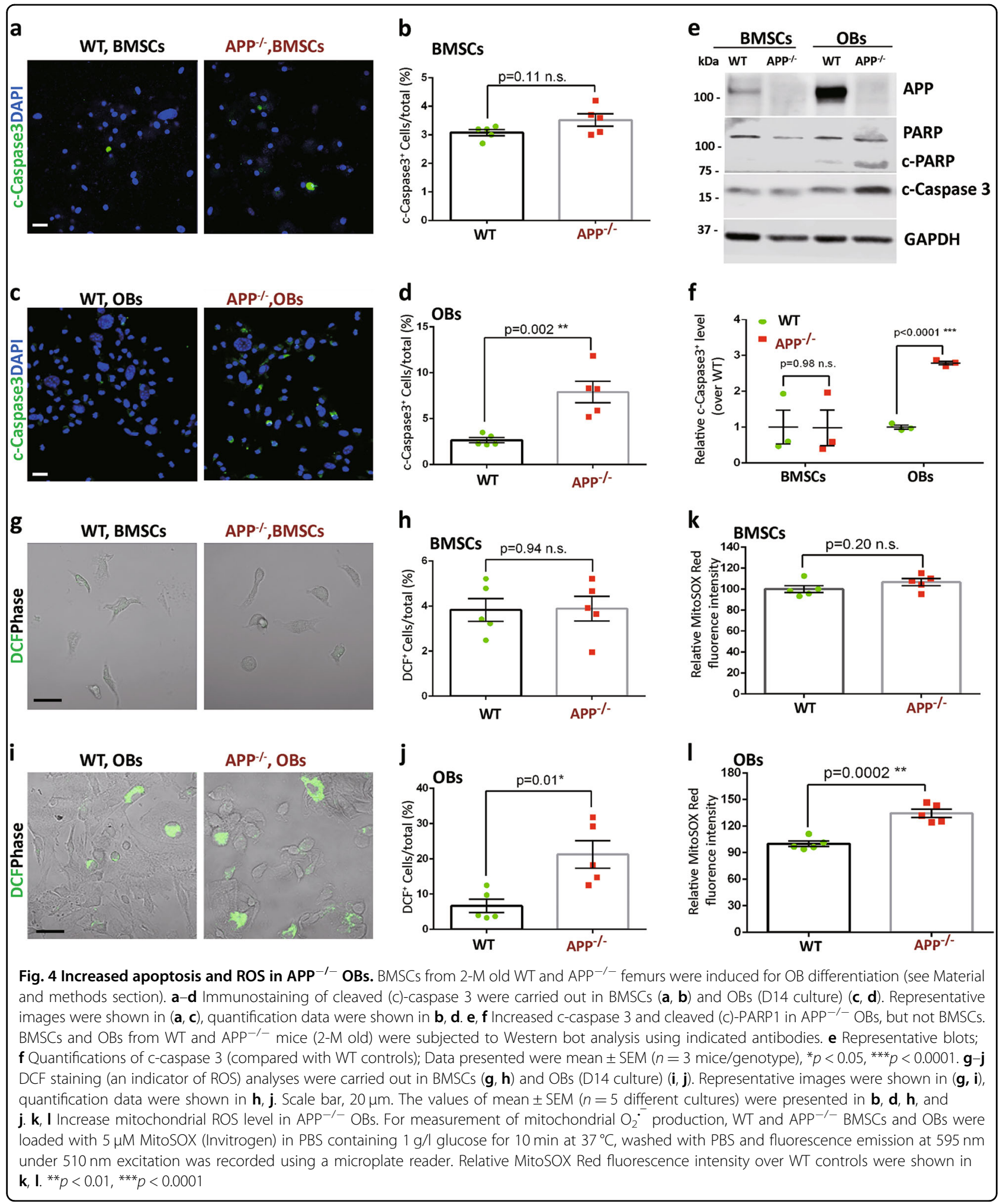

OBs than those in control OBs but not BMSCs (Fig. 5e-j), suggesting mitochondrial fragmentation in $\mathrm{APP}^{-1-} \mathrm{OBs}$. This view was further supported by the observation of a decrease of mitofusin 2 (Mfn2), a GTPase critical for mitochondrial fusion, in mitochondrial fractions of $\mathrm{APP}^{-1-}$ OBs (Fig. 5a, d). These results suggest that APP 

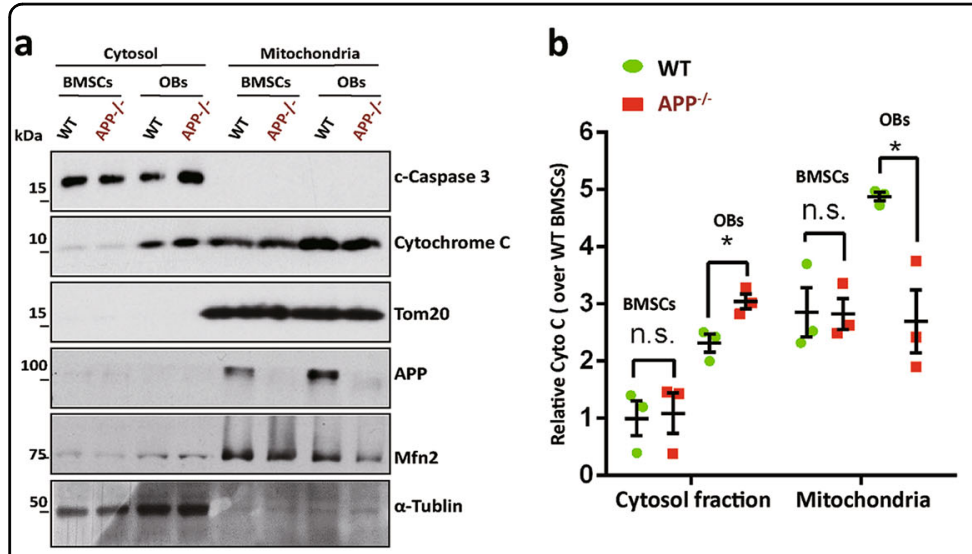

\section{c}

WT

a APP $\%$

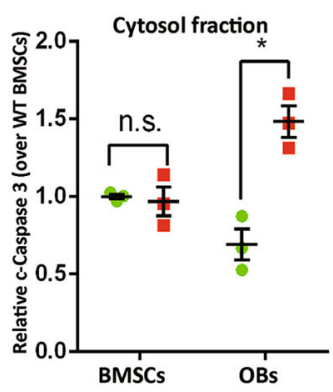

d

d
WT
APP $\%$

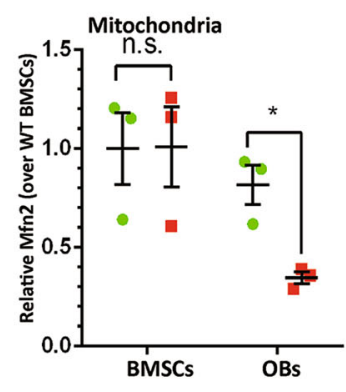

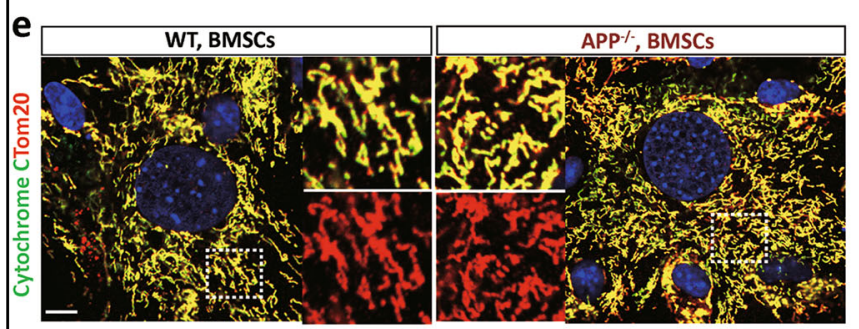
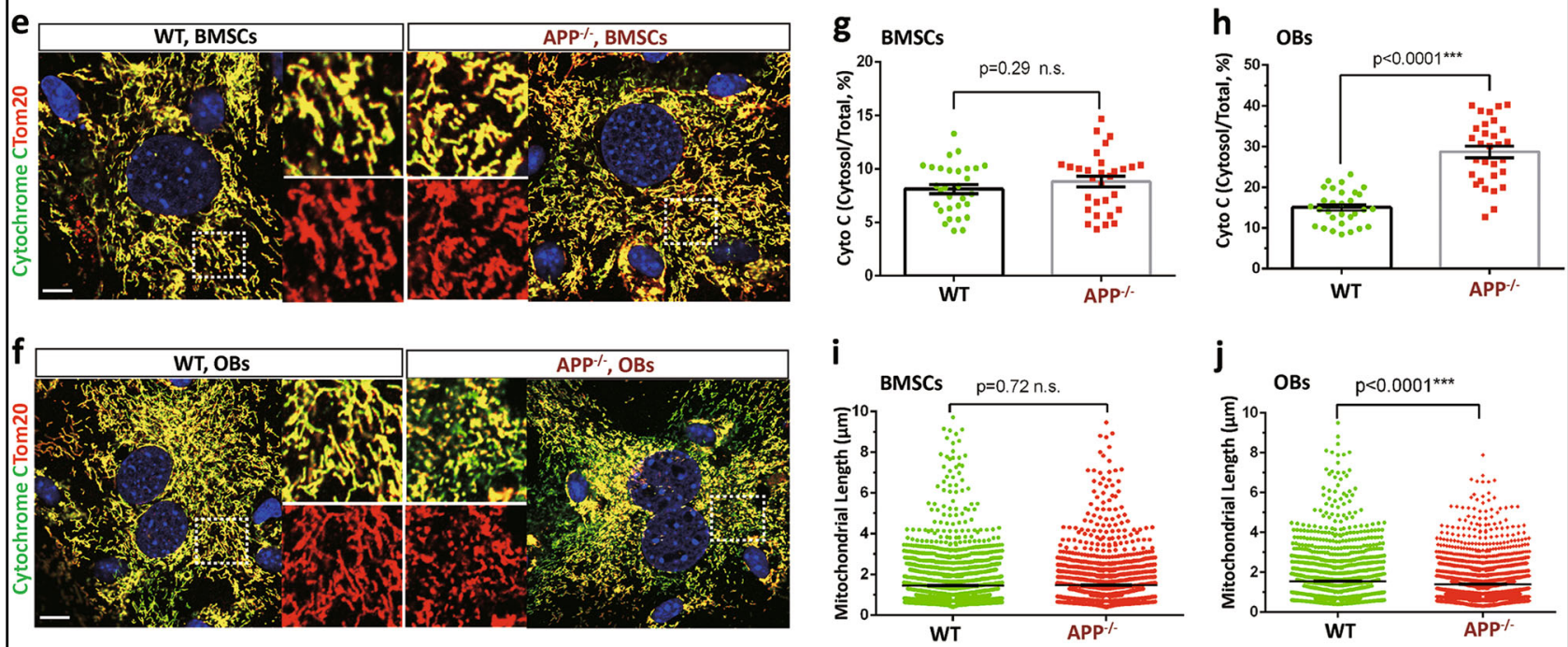

Fig. 5 Increased cytochrome C release and mitochondrial fragmentation in APP ${ }^{-1-}$ OBs, but not BMSCs. a-d Reduced mitochondrial cytochrome $\mathrm{C}$ and Mfn2 in APP ${ }^{-1-}$ OBs, but not BMSCs. Mitochondrial and cytosol fractions of BMSCs and OBs from WT and APP ${ }^{-1-}$ mice (2-M old) were subjected to Western bot analysis. a, Representative blots; b-d Quantifications of protein levels (compared with WT BMSCs); Data presented were mean \pm SEM ( $n=3$ mice/genotype); ${ }^{*} p<0.05$. $\mathbf{e}-\mathbf{j}$ Increased cytosolic cytochrome $C$ and mitochondrial fragmentation in APP ${ }^{-/-} \mathrm{OBs}$, but not BMSCs. OBs (D14 culture) were in vitro differentiated from BMSCs from WT and APP ${ }^{-1-}$ mice (2-M old). Both BMSCs and OBs were co-immunostained with indicated antibodies. Mitochondria were labeled by Tom20. Representative images were shown in $\mathbf{e}$, f. Higher power views of mitochondria (Tom20) and cytochrome $C$ were also included in $\mathbf{e}, \mathbf{f}$ Scale bar, $5 \mu \mathrm{m} . \mathbf{g}, \mathbf{h}$ Quantifications of cytochrome $\mathrm{C}$ fluorescence in cytosol over total. The cytosolic cytochrome $\mathrm{C}$ was defined by cytochrome $\mathrm{C}^{+}$Tom $20^{-}$staining. Data presented were mean \pm SEM ( $n=30$ cells from 3-different cultures). $\mathbf{i}, \mathbf{j}$ Quantifications of mitochondrial length. Shown were grouped column scatter (with mean \pm SEM). $n=1500-2000$ mitochondria from 30 different cells of each group. ${ }^{* *} p<0.0001$

in OBs may be required for mitochondrial fusion dynamics.

\section{Mitochondrial defects in APP ${ }^{-1-}-$ BM-OB $^{-}$cultures}

Mitochondria are the major source of ROS and ATP production, which are increased during $\mathrm{OB}$ genesis ${ }^{22}$. To understand how APP prevents ROS, we examined the following mitochondrial proteins, in addition to Mfn2, in WT and $\mathrm{APP}^{-1-}$ BMSCs and OBs. Sirt3 (a mitochondrial deacetylase Sirtuin 3) and Sod2 (superoxide dismutase 2) are both critical proteins for anti-oxidant response $\mathrm{r}^{23,24}$ and OB-mediated bone formation ${ }^{22}$. Drp1 (dynamin- related protein 1) is a key protein for mitochondrial fis$\operatorname{sion}^{25-28}$. Mitochondrial complex proteins (I, II, III, IV, and $\mathrm{V}$ ) are essential for ATP production from ETC (electoral transfer chain) reaction. Remarkably, Sirt3, Sod2, Mfn2, but not Drp1 or Tom20, were reduced in $\mathrm{APP}^{-1-} \mathrm{OBs}$, but not BMSCs (Fig. 6a, b). In addition, the complex I (subunit UQCRC1) and V, but not II (subunit SDHA), III and IV, were also lower in $\mathrm{APP}^{-/-} \mathrm{OBs}$ (Fig. 6a, b). These results suggest a reduction in Sirt3/ Sod2 mediated anti-oxidant response and impairments in mitochondrial fusion and ETC function in $\mathrm{APP}^{-/-} \mathrm{OBs}$, which are likely to contribute to the increase of ROS. 
a

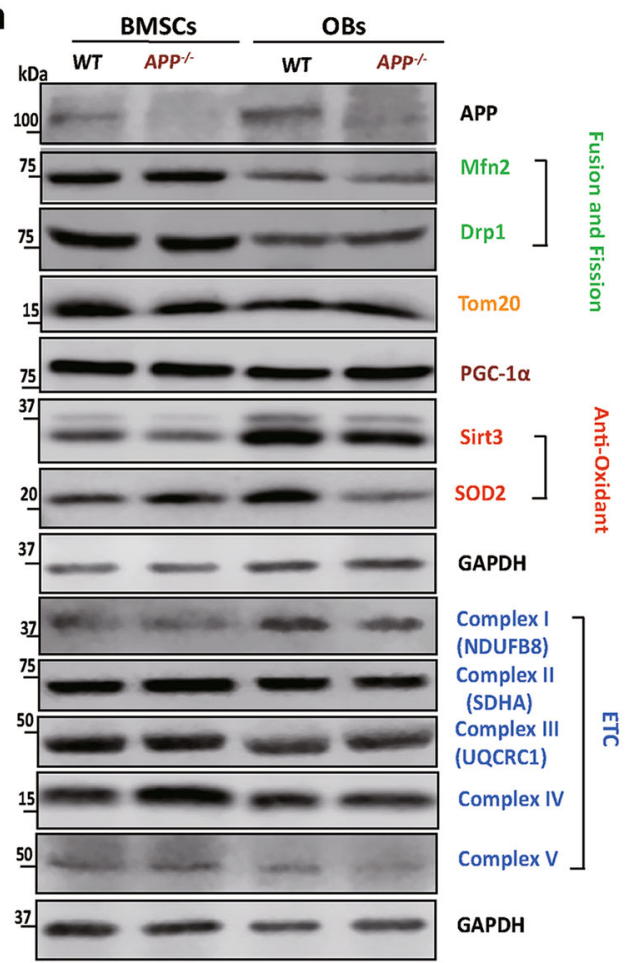

C

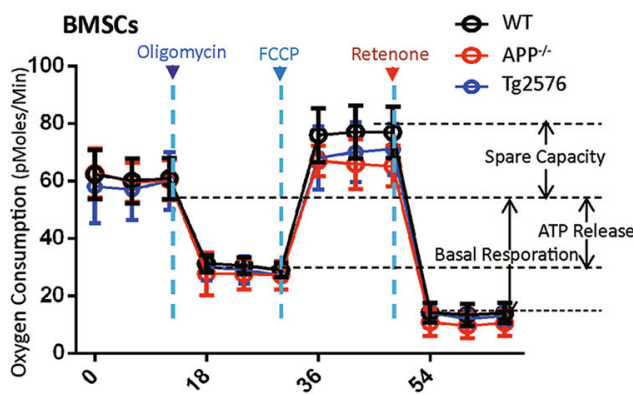

e

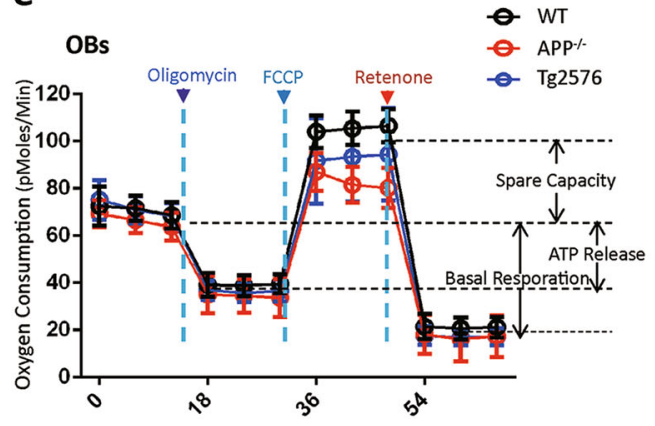

b

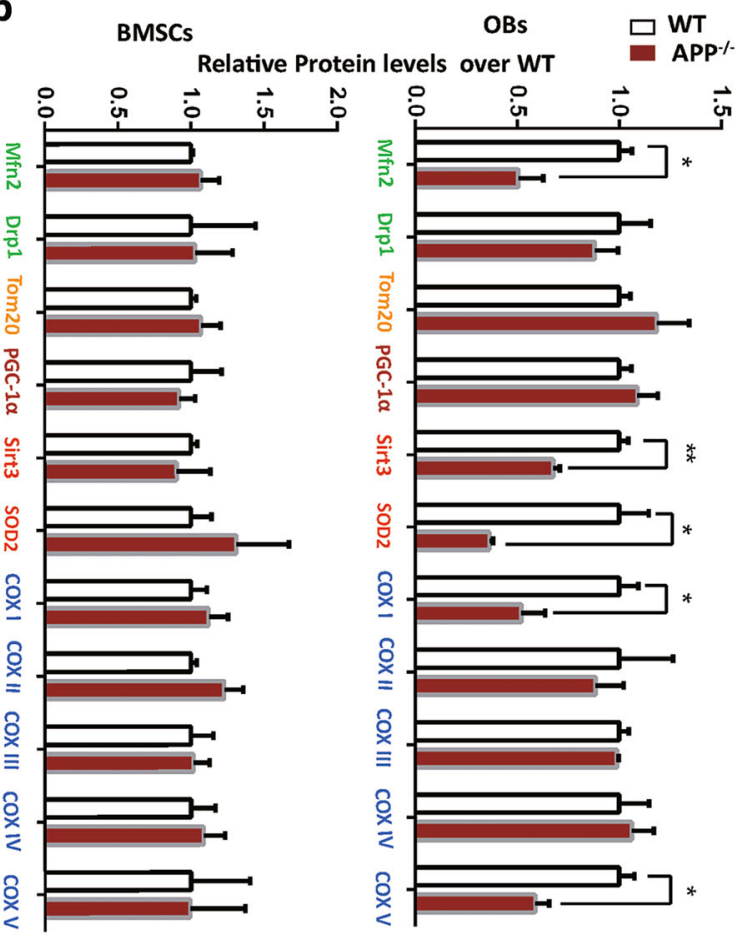

d

BMSCs
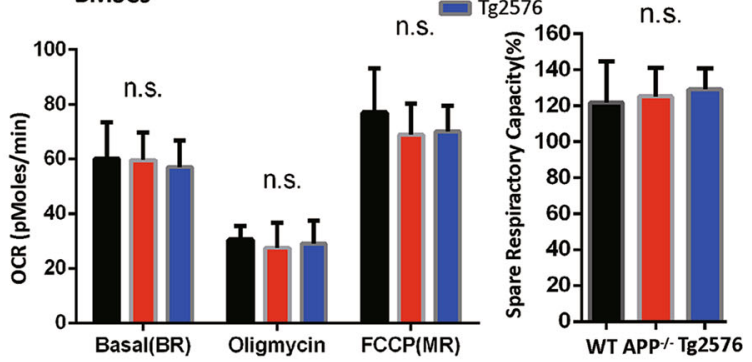

f
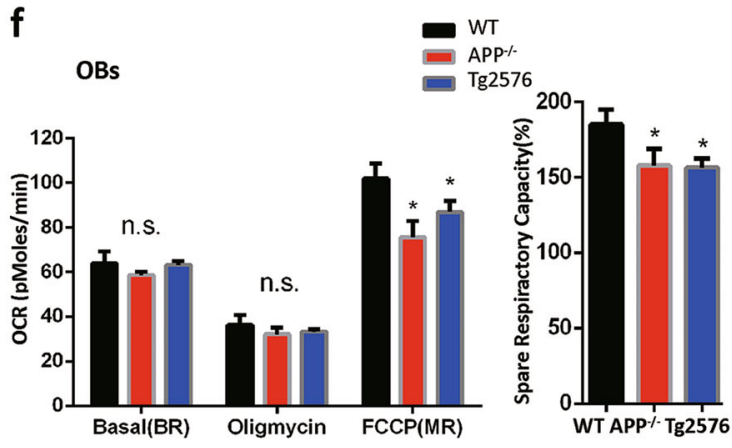

Fig. 6 Mitochondrial defects in APP ${ }^{-1-}-$ BM-OB cultures. $\mathbf{a}, \mathbf{b}$ Western blot analysis of mitochondrial proteins using indicated antibodies. a Representative blots; $\mathbf{b}$ Quantifications of protein changes in APP ${ }^{-1-}$ mice (compared with WT); Data presented were mean \pm SEM $(n=3$ mice/ genotype); ${ }^{*} p<0.05,{ }^{* *} p<0.01$. c-f Mitochondrial functional deficits in APP ${ }^{-1-}$ and Tg2576-OBs, but not their BMSCs, by a Seahorse XF96 analyzer. OBs (D14 culture) were in vitro differentiated from BMSCs of 2-M old WT, APP ${ }^{-1-}$, or Tg2576 femurs. Both BMSCs (c, d) and OBs (e, f) were subjected to the measurements of the real-time $\mathrm{O}_{2}$ consumption rate (OCR) by the Seahorse XF96 analyzer. In c-f, to validate the OCR measurement, ATP synthase inhibitor, oligomycin $(2 \mu \mathrm{M})$, was treated after recording the basal line, and the pharmaceutical uncoupler, FCCP $(3 \mu \mathrm{M})$, and the Complex I inhibitor rotenone $(1 \mu \mathrm{M})$ were then treated as indicated. Representative traces of OCR were shown in $\mathbf{c}, \mathbf{e}$. Quantifications were presented in $\mathbf{d}, \mathbf{f}$. The spare respiratory capacity changes (compared with BR) were also showed in the right columns of $d$, f. The values of mean \pm SEM $(n=3)$ were shown. ${ }^{*} p<0.05$ 
Considering the importance for PGC-1alpha for mitochondria biogenesis and function, PGC1alpha was also examined. It was comparable in $\mathrm{APP}^{-1-}$ OBs or BMSCs to that in WT OBs/BMSCs (Fig. 6a, b), suggesting little role, if there is any, that APP regulates PGC1 $\alpha$ expression.

We next examined mitochondrial function (respiratory capacity) in $\mathrm{APP}^{-1-}$ BMSCs and OBs by the Seahorse plate form assay to real time measure oxygen $\left(\mathrm{O}_{2}\right)$ consumption rates (OCR). No difference in rates of the basal oxygen consumption (BR) or oxygen consumption (OCR) was detected between WT and $\mathrm{APP}^{-1-}$ OBs (Fig. 6e, f). But, the maximal respiratory rate (MR) induced by mitochondrial un-coupler (Carbonyl cyanide-ptrifluoromethoxyphenylhydrazone (FCCP)) treatment was reduced in $\mathrm{APP}^{-1-} \mathrm{OBs}$ (Fig. 6e, f), resulting in a deficiency in spare respiratory capacity (SR) (Fig. 6f). Again, this deficit was detected only in $\mathrm{APP}^{-1-} \mathrm{OBs}$, but not in $\mathrm{APP}^{-1-}$ BMSCs (Fig. 6c, d), demonstrating dysfunctional mitochondria in cultured $\mathrm{APP}^{-1-} \mathrm{OBs}$.

As similar osteoporotic deficits were observed in $\operatorname{Tg} 2576$ mice $^{14,15}$, we asked if OBs from Tg2576 mice show any mitochondrial deficit as that of $\mathrm{APP}^{-1-}$ OBs. Indeed, the SR was lower in OBs from Tg2576 mice (Fig. 6e, f), supporting the view for APPswe acting as a dominant negative regulator in this event.

In addition to mitochondrial function in ATP production, we examined glycolysis, an oxygen independent pathway for ATP production, in WT and $\mathrm{APP}^{-1-}$ BMSCs and OBs (Fig. S5). The ATP level by the glycolytic pathway, but not mitochondrial oxidative phosphorylation (OXPHO)-pathway, was reduced in $\mathrm{APP}^{-1-}$ BMSCs (Fig. S5a-b), suggesting a deficit in glycolysis. However, in $\mathrm{APP}^{-1-}$ OBs, whereas OXPHOSdependent ATP production was reduced (Fig. S5c), the glycolysis regulated ATP level was increased (Fig. S5d), in line with the reports that blocking OXPHOS pathway results in a compensatory increase of ATP production by the glycolytic activation ${ }^{29,30}$. The reduction of OXPHOS-dependent ATP production in $\mathrm{APP}^{-1-} \mathrm{OBs}$ was in line with the results from the Seahorse assay. Together, these results demonstrate an impaired oxygen or mitochondrial dependent ATP production in $\mathrm{APP}^{-1-}$ OBs, but not BMSCs.

\section{Increased mitophage/autophage in $\mathrm{APP}^{-1-}$ OBs and APP- KO MC3T3 cells}

In addition to mitochondrial fission, reduced Mfn2 is frequently associated with increased mitophage, a critical pathway for mitochondrial degradation/removal by autophage $^{31}$. We thus examined LC3-II and P62/ SQSTM1, both autophagic proteins, in $\mathrm{APP}^{-1-}$ BMSCs and OBs (Fig. 7a, b). Indeed, both LC3-II and P62 were elevated in $\mathrm{APP}^{-1-}$ OBs, but not BMSCs (Fig. 7a, b), implicating an increase in mitophage.
To further test this view, we generated APP-KO MC3T3 cells (an OB cell line) by Crispr-Cas9 method (Fig. 7a) and examined LC3-II and P62 levels in these cells by both Western blot and immunostaining analyses. Remarkably, both LC3-II and P62 were higher in differentiated APP-KO MC3T3 cells than those of control cells (Fig. 7a, b). Immunostaining analysis of MC3T3 cells expressing exogenous GFP-LC3 showed enlarged $\mathrm{LC}^{+}$vesicles in APP-KO MC3T3 cells (Fig. 7c, d). Interestingly, the endogenous P62 was increased not only in APP-KO cells (compared with controls), but also in GFP-LC3 expressing cells (compared with un-transfected cells) (Fig. 7c, e). P62 mRNA levels were also higher in $\mathrm{APP}^{-1-}$ OBs than that of WT controls (Fig. 7f). Furthermore, MC3T3 cells expressing exogenous GFP-LC3 and Mito-RFP displayed more co-localization of GFP-LC3 with Mito-RFP in APP-KO cells than that of control cells (Fig. S6a-c). However, MC3T3 cells expressing LC3-eGFP-mCherry fusion protein showed comparable ratio of $\mathrm{GFP} / \mathrm{mCherry}$ between control and APP-KO cells (Fig. 7g, h), suggesting little role of APP in autophagic-lysosome fusion or autophagic flux. Together, these results support the view for an increased mitophage/autophage formation in $\mathrm{APP}^{-1-} \mathrm{OBs}$.

Mfn2 is also believed to be an element for the maintenance of a correct mitochondria-associated-ER membrane (ER-MAM) juxstapostition ${ }^{32,33}$. We measured mitochondrial-ER contact sites by imaging and quantification analysis of the co-localization of Mito-RFP (a mitochondrial marker) with Sec61b-GFP (an ER marker). No significant difference was detected between WT and APP $^{-1-}$ OBs (Fig. S6d-f).

\section{Diminished deficits in mitochondrial function, $O B-$ differentiation, and bone homeostasis in $\mathrm{APP}^{-1-} \mathrm{OBs}$ and mice treated with NAC}

Increased ROS is believed to be detrimental for $\mathrm{AD}$ pathogenesis $^{34}$. Both AD-like and skeletal ageing-like phenotypes in Tg2576 mice can be ameliorated by treatments with anti-oxidant(s), NAC, a glutathione (GSH) precursor that increases GSH levels and reduces free radicals $^{15,35}$. The similar osteoporotic and mitochondrial deficits between $\mathrm{APP}^{-/-}$and Tg2576 mice lead us to ask if NAC could also diminish the bone deficits in $\mathrm{APP}^{-1-}$ mice. To this end, we first tested NAC's effect on mitochondrial and OB-differentiation deficits in cultured $\mathrm{APP}^{-1-} \mathrm{OBs}$. As expected, the impaired spare capacity of mitochondria of $\mathrm{APP}^{-1-}$ OBs was diminished when NAC was present in the $\mathrm{OB}$ cultures (Fig. 8a, b). NAC increased ALP positive $\mathrm{OBs}$ from $\mathrm{APP}^{-1-} \mathrm{BMSC}$ cultures, partially restoring the OB-differentiation (Fig. 8c, d).

We next tested NAC's effect in vivo. $\mathrm{APP}^{-1-}$ mice (at age of 1-2 $\mathrm{M}$ old) were fed with drinking water 


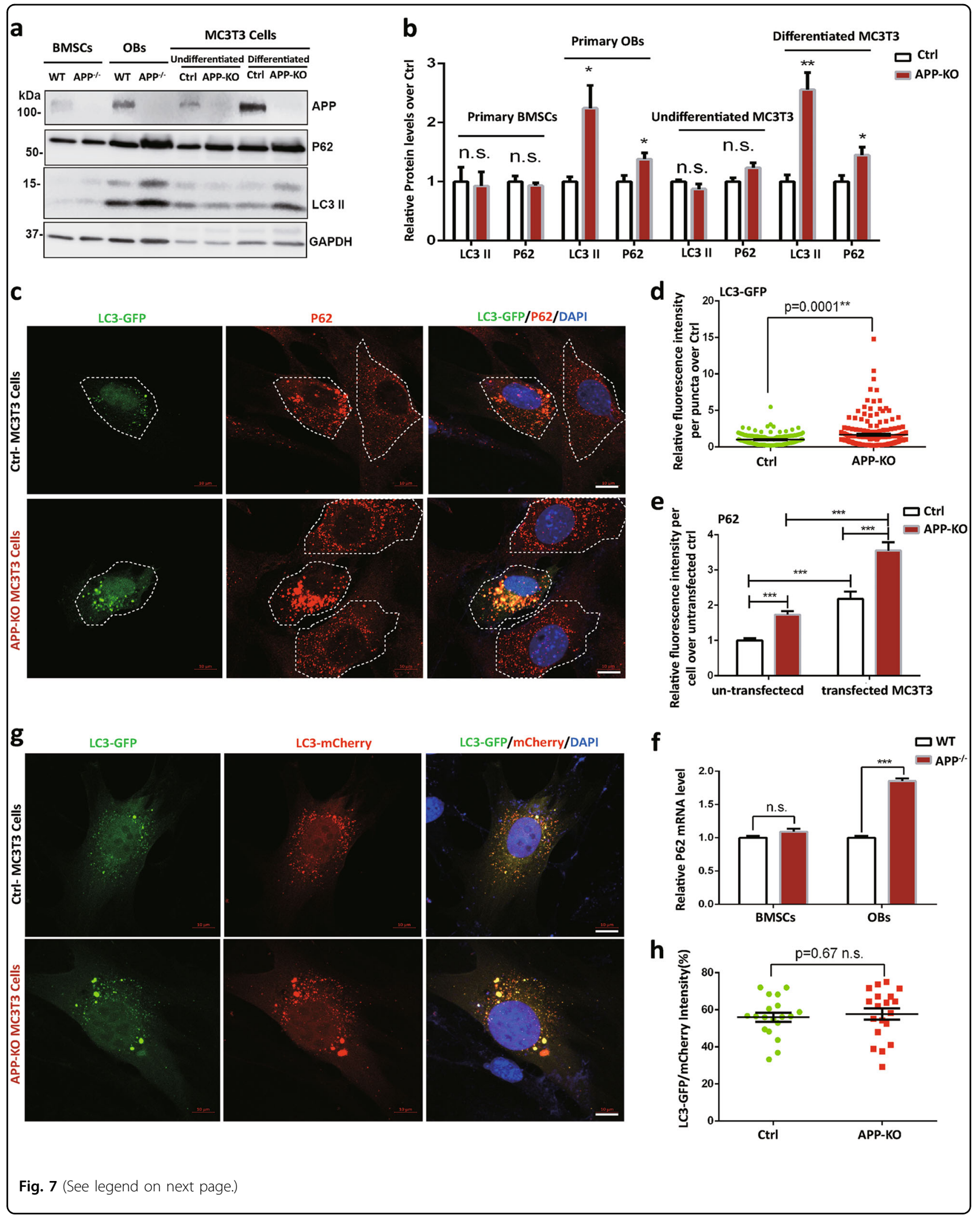


(see figure on previous page)

Fig. 7 Increased LC3 II and P62 proteins in APP ${ }^{-1-}$ OBs and differentiated APP-KO MC3T3 cells. a, b Western blot analysis of proteins using indicated antibodies. a Representative blots; $\mathbf{b}$ Quantifications of relative protein levels. Data presented were mean $\pm \operatorname{SEM}(n=3)$; ${ }^{*} p<0.05$. c-e Increased LC3 and P62 fluorescence intensities in differentiated APP-KO MC3T3 cells. To differentiate pre-osteobalstic MC3T3 cells to mature osteoblastic MC3T3 cells, both control and APP-KO MC3T3 cells were induced by the addition of $50 \mathrm{ng} / \mathrm{ml}$ ascorbic acid and $10 \mathrm{mM} \beta$ glycerophosphate to standard growth medium for 7 days. Then those differentiated cells were transfected with GFP-LC3 plasmid. Seventy-two hours after transfection, cells were fixed and immunstained with indicated antibodies. c Representative images. Scale bar, $10 \mu m$; d, e quantification analyses of fluorescence intensity of LC3-GFP puncta (d) and endogenous P62 per cell in un-transfected and transfected MC3T3 cells (e). Data presented were mean \pm SEM, $n=20$ from three-different cultures, ${ }^{* *} p<0.01,{ }^{* * *} p<0.0001$. f RT-PCR analysis of P62 mRNA levels in WT and APP ${ }^{-\prime-}$ BMSCs and OBs. Data presented were mean \pm SEM $(n=3) ;{ }^{* * *} p<0.0001 . \mathbf{g}$, h Normal autophagosome-lysosome fusion in APP-KO MC3T3 cells. Differentiated control and APP-KO MC3T3 Cells were transfected with LC3-mCherry-GFP fusion plasmid. Three days after transfection, Cells were then subjected to fluorescence imaging analysis. $\mathbf{g}$ Representative images. Scale bar, $10 \mu \mathrm{m} ; \mathbf{h}$ Quantification analysis. Data presented were ratios of GFP/ mCherry intensities, mean $\pm \operatorname{SEM}(n=18) .{ }^{*} p<0.05$

containing vehicle or NAC $(2 \mathrm{mg} / \mathrm{kg} /$ day $)$ for $3-\mathrm{M}$ and their femur bone and sera samples were then collected for phenotypic analysis. As shown in Fig. 8e, f, bone volume, particular trabecular bone volume, was increased in NAC-treated $\mathrm{APP}^{-/-}$femurs, compared with that of vehicle treatment, based on $\mu \mathrm{CT}$ analysis. Bone formations, viewed by Goldner's trichrome staining of osteoid density and by measurement of serum levels of osteocalcin, were also elevated in NACtreated $\mathrm{APP}^{-1-}$ mice (Fig. $8 \mathrm{~g}-\mathrm{i}$ ). These results thus demonstrate an efficient amelioration of the osteoporotic deficit by NAC. In addition to NAC's effect on bone formation, we examined its role in bone resorption in WT and $\mathrm{APP}^{-1-}$ mice. Bone resorption, indicated by serum levels of PYD, was reduced by NAC treatment of WT, but not $\mathrm{APP}^{-1-}$, mice (Fig. 8j). Taken together, these results suggest that NAC's amelioration of the osteoporotic deficit in $\mathrm{APP}^{-/-}$mice may be due in large to its anti-oxidant effects on OBs.

\section{Discussion}

This study provides evidence for APP to be necessary for $\mathrm{OB}$ survival and bone formation, and thus maintaining bone homeostasis. Mechanistically, the osteoblastic APP appears to be critical for mitochondrial function, and thus preventing from cytochrome $\mathrm{C}$ release, ROS production, and apoptosis.

APP regulation of bone homeostasis is evident based on $\mu \mathrm{CT}$ and histological analyses of long bone structures of $\mathrm{APP}^{-1-}$ mice. This function appears to be largely due to APP-promotion of OB-mediated bone formation. Interestingly, there is a remarkable similarity in bone deficits between $\mathrm{APP}^{-1-}$ and Tg2576 mice. Both show decreases in OB-genesis, bone formation, and bone mass, and transiently increases in $\mathrm{OC}$-genesis and bone resorption in young age. These observations implicate that APPswe may act as a dominant negative regulator of endogenous APP in both OB and OC cells.

How does APP regulate OB-function? We posit that APP enhances OB-function likely by promoting $\mathrm{OB}$ mitochondrial function, preventing ROS production and cytochrome $\mathrm{C}$ release, and thus increasing $\mathrm{OB}$ survival, in light of the following observations. First, the early stage of $\mathrm{OB}$ differentiation from BMSCs and $\mathrm{OB}$ progenitor proliferation appeared to be normal in $\mathrm{APP}^{-/-}$cells (Fig. S3cd); however, the late stage of $\mathrm{OB}$ differentiation and function, which depend on OB-survival, were severally impaired in $\mathrm{APP}^{-1-} \mathrm{OB}$ cultures (Fig. 3). Second, OB cell death markers, including cleaved caspase 3, cleaved PARP1, cytochrome $\mathrm{C}$ release, and ROS, were all elevated in $\mathrm{APP}^{-1}$ OBs, but not BMSCs (Figs. 4, 5). Third, mitochondrial morphological and functional deficits in $\mathrm{APP}^{-1-} \mathrm{OBs}$, but not BMSCs, were evident. Critical proteins for anti-oxidant response (e.g., SIRT3 and SOD2) and proteins for mitochondrial ETC functions (e.g., complex I, and V) were all reduced in $\mathrm{APP}^{-1-} \mathrm{OBs}$ (Fig. 6a, b); mitochondrial SR (viewed by Seahorse plate form assay) and OXPHOSdependent ATP production were much lower in $\mathrm{APP}^{-1-}$ OBs (Fig. 6e, f, Fig. S5); and mitochondrial fusion (viewed by immunostaining analysis of its morphology and western blot analysis of MFN2) was also impaired in $\mathrm{APP}^{-1-} \mathrm{OBs}$ (Figs. 5 and 6a, b). Finally, treatments with anti-oxidant $\mathrm{NAC}$ in $\mathrm{APP}^{-1-} \mathrm{BMSC}-\mathrm{OB}$ cultures restore mitochondrial function and OB-differentiation (Fig. 8a-d), and NAC treatment in $\mathrm{APP}^{-1-}$ mice increased bone formation and diminished the osteoporotic deficit (Fig. 8c-j).

APP regulation of mitochondrial function is also supported by the following observations. It is largely distributed in mitochondria ${ }^{21}$ (Fig. 5a and Fig. S4); $\mathrm{A} \beta$ disrupts mitochondrial fusion and function ${ }^{36}$; and over expression of APPswe in various cell lines results in mitochondrial fusion deficits (data not shown). Why mitochondrial function is so important for OB-mediated bone formation? Notice that osteogenic induction is accompanied by robust oxygen consumption; and mitochondrial biogenesis is increased during OB-differentiation ${ }^{22}$. In line with this view are our observations of much higher levels of mitochondrial antioxidant (Sirt3 and Sod2) and Cytochrome $\mathrm{C}$ proteins in OBs than those in BMSCs (Figs. 5a, b and 6a); and APP was also higher in OBs than that in BMSCs (Figs. 5a and 6a). 


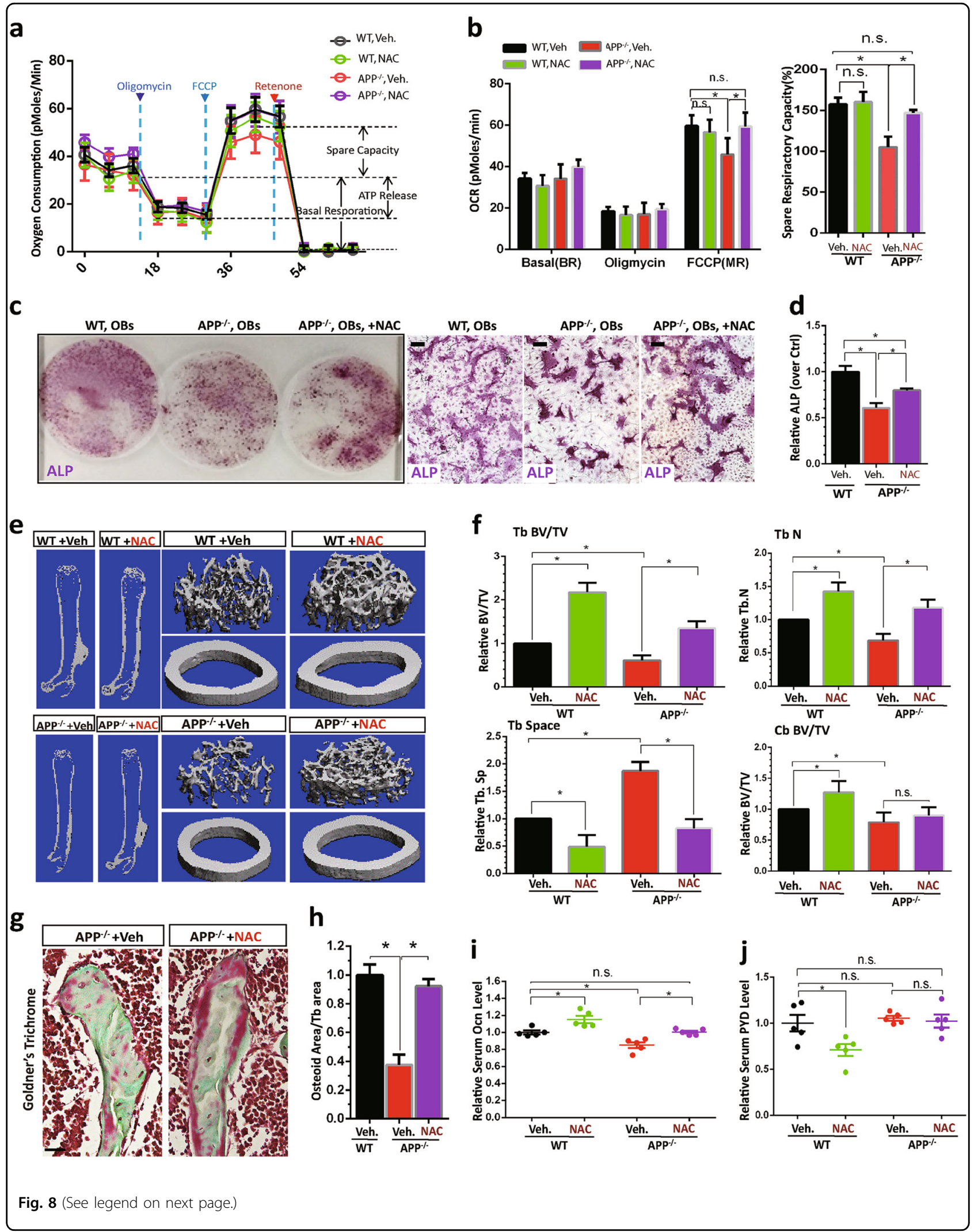


(see figure on previous page)

Fig. 8 Diminished deficits in mitochondrial function, OB-differentiation, and bone homeostasis in APP ${ }^{-/-}$OBs and mice treated with NAC. a, b NAC restore of mitochondrial function of APP ${ }^{-1-}$ OBs. OBs (D1 culture) were treated with NAC (1 mM, overnight) or vehicle (veh) control. At D14, OBs' mitochondrial function was evaluated by a Seahorse XF96 analyzer. Representative traces of OCR were shown in a Quantifications were presented in $\mathbf{b}$. The values of mean \pm SEM $(n=3)$ were shown. ${ }^{*} p<0.05$. $\mathbf{c}$, d NAC restore of OB differentiation (viewed by ALP staining) from BMSCs of $\mathrm{APP}^{-1-}$ mice. As $\mathbf{a}, \mathbf{b}, \mathrm{D} 1-\mathrm{OB}$ cultures were treated with NAC $(1 \mathrm{mM})$ or veh control, and D14-OBs were stained with ALP. Representative images were shown in c, scale bar $20 \mu \mathrm{m}$, and the quantification analysis of the average ALP activities (normalized by WT control) was presented in $\mathbf{d}$. The values of mean \pm SEM from three different cultures were presented. ${ }^{*} p<0.05$. e-j APP ${ }^{-/}$mice (at age of 1-M old, 5 per group, male) were fed with drinking water containing with vehicle (Veh.) or NAC (2 mg/kg/day) for 3 months, and their femur bone samples and sera were collected for phenotypic analyses. e, $\mathbf{f}$ The $\mu \mathrm{CT}$ analysis displayed an increased trabecular bone volumes of NAC-treated APP ${ }^{-1-}$ femurs. The trabecular bone thickness (Tb.Th) and trabecular separation (Tb.Sp) were all ameliorated or improved by NAC treatment. The cortical bone volumes were unaffected by NAC. Scale bar, 1 mm. $\mathbf{g}$, h Goldner's trichrome staining analysis showed an increased osteoid numbers of trabecular bones in NAC-treated APP ${ }^{-\prime}$

- femurs. Representative images were shown in $\mathbf{g}$, scale bar, $20 \mu \mathrm{m}$, and the data were quantified (osteoid area/bone area, normalized by WT) and showed in $\mathbf{h}$. $\mathbf{i}$ Increased serum levels of osteocalcin in NAC-treated APP ${ }^{-1-}$ mice were detected by ELISA assays. $\mathbf{j}$ No change of serum levels of PYD in NAC-treated $\mathrm{APP}^{-/-}$mice. In $\mathbf{f}, \mathbf{h}-\mathbf{j}$, the values of mean \pm SEM from five different animals per genotype were shown. ${ }^{*} p<0.05$

The increased autophagic proteins, LC3-II and P62, in $\mathrm{APP}^{-1-}$ OBs (Fig. 7a, b) suggest a role that APP may play in autophagosome formation. Considering no obvious deficits in mitochondrial protein degradation (Fig. 6a, b) and autophage-lysosome fusion (Fig. $7 \mathrm{~g}, \mathrm{~h}$ ) in $\mathrm{APP}^{-1-}$ OBs, the increased P62 is thus likely due to LC3-mediated autophagosome formation and ROS-induced P62 expression $^{37,38}$. This view is also supported by the observations that expressing GFP-LC3 in MC3T3 cells increased endogenous P62 (Fig. 7c-e) and P62 transcripts are increased in $\mathrm{APP}^{-1-}$ OBs (Fig. 7f).

How does APP regulate OB mitochondrial function? APP in mitochondrial membrane may play a role in maintaining the stability of Mfn2 and Sirt3/Sod2, promoting Mfn2mediated mitochondrial fusion and function and Sirt3/Sod2 mediated anti-oxidant response, and thus preventing ROS production, an inducer of $\mathrm{OB}$ apoptosis and an inhibitor of OB differentiation and function. Additionally, APP in mitochondrial membrane may prevent Cytochrome $\mathrm{C}$ release from mitochondria, and thus inhibiting caspase 3driven $\mathrm{OB}$ apoptosis. This view is in agreement with the observations that treatment with NAC ameliorates the osteoporotic deficit not only in young adult Tg2576 mice, but also in $\mathrm{APP}^{-1-}$ mice. Interestingly, NAC pretreatment in $\mathrm{AD}$ animal models, including $\mathrm{Tg} 2576$ mice, reduces $\mathrm{A} \beta$ levels, decreases lipid peroxidation, and improves cognition $^{39}$. In light of these results, we speculate that NAC may be an effective therapy in amelioration of $\mathrm{AD}$-associated bone deficit.

\section{Materials and methods}

\section{Reagents and animals}

Antibodies including APP (\#2452, rabbit polyclonal), Cleaved Caspase-3(\#9664), Mitofusin-2(Mfn2) (\#9482), cytochrome C (\#4272 Rabbit polyclonal), PARP1(\#9452) and SirT3 (Rabbit mAb \#5490) were purchased from Cell Signaling Technology (Danvers, MA, USA), Ki67
(AB9260) was purchase from EMD Millipore, DRP1 (NB110-55288) was purchased from Novus Biologicals, Cytochrome C (556432, mouse monoclonal) was purchase from BD Bioscience,Tom20(PA5-52843, Rabbit polyclonal), Complex I(NDUFB8, \#459210), Complex II (SDHA, \#459200), Complex III (UQCRC1, \#459140), Complex IV(A21348) and Complex V(ATP synthase beta, A21351) monoclonal antibodies were purchased from ThermoFisher, PGC-1 $\alpha(\mathrm{ab} 54481), \quad$ SOD2 (ab13533), P62(ab56416) and LC3 A/B (ab128025) were purchased from Abcam, Tom20 (sc-17764, mouse monoclonal), GAPDH, $\beta$-actin and $\alpha$-tublin were purchased from Santa Cruz. H2DCFDA (D399) and MitoSOX Red (M36008) was purchased from Thermofisher Scientific (Carlsbad, CA, USA), N-acetyle-L-cysteine (NAC) were purchased from Sigma (St. Louis, MO, USA).

$\mathrm{APP}^{-1-}$ mice (stock number 004133, C57BL/6 background) were purchased from the Jackson's laboratory (Bar Harbor, ME, USA, USA). Same aged wild type (WT) mice (C57BL/6 background) were used as controls. Male and female mice were characterized. Ocn-Cre; Ai9 (Ocn; $\mathrm{Td})$ and $\mathrm{APP}^{-1-}$; Ocn; $\mathrm{Ai} 9$ mice were generated by crossing Ai9 mice (from the Jackson Laboratory, donated by Dr. Hongkui Zeng, Allen Institute for Brain Science) with Ocn-Cre transgenic mice (provided by Dr. T. Clemens, Johns Hopkins Medical School) and $\mathrm{APP}^{-1-}$ mice. Ai9 mice have a loxP-flanked STOP cassette preventing translation of a CAG promoter-driven red fluorescent protein variant (tdTomato). All mice were housed in a room with a $12 \mathrm{~h}$ light/dark cycle with ad libitum access to water and rodent chow diet (Diet 7097, Harlan Teklad). All experimental procedures were approved by the IACUC (Institutional Animal Care and Use Committee) at the Case Western Reserve University and Augusta University, in accordance US National Institutes of Health guidelines. 


\section{In vitro $\mathrm{BMSC}, \mathrm{OB}$, and adipocyte cultures}

The primary cultures of BMSCs, OBs, and adipocytes were carried out as described previously ${ }^{15,40}$. In brief, the whole bone marrow cells flushed out from long bones of 2-3 month old WT, $\mathrm{APP}^{-1-}$ mice with DMEM were filtered through a 70-mm filter mesh, washed, re-suspended, and then plated in 100-mm dishes with growth medium (DMEM plus 10\% FBS), which were incubated at $37^{\circ} \mathrm{C}$ with $5 \% \mathrm{CO}_{2}$. The non-adherent cells were removed $72 \mathrm{~h}$ after medium changing. The attached $\mathrm{BM}$ cells were cultured with the growth medium for 7 days. These cells were then resuspended and plated at a density of 12-well plates or culture dishes, and cultured for another 3-6 days with the same growth medium. These cells, so called BMSCs, were used for Western blot, immune-staining analysis, or subjected to further differentiation with $\mathrm{OB}$ or adipocyte differentiation medium. For OBs and adipocytes, BMSCs were plated in densities of $1 \times 10^{4} / \mathrm{cm}^{2}$ and $3 \times 10^{4} / \mathrm{cm}^{2}$ at 12 -well plates for $\mathrm{OB}$ and adipocyte inductions, respectively. To induce $\mathrm{OB}$ genesis, BMSCs were exposed in osteogenic medium (DMEM containing 10\% FBS, $1 \% \mathrm{P} / \mathrm{S}, 10 \mathrm{mM} \quad \beta$ glycerophosphate and $50 \mu \mathrm{M}$ L-ascorbic acid-2- phosphate). After 7-14 days, ALP (alkyline phosphatase) staining and quantification were performed as described previously ${ }^{15,40,41}$. At D14, the OBs were used Western blot, immune-staining analysis. After 21 days, to visualize calcified matrix, Alizarin Red S staining were performed and quantification as previous described ${ }^{40}$.

To induce adipogenesis, cells were maintained to adipocyte differentiation media (DMEM containing $10 \%$ FBS, $1 \% \mathrm{P} / \mathrm{S}, 0.5 \mathrm{mM}$ isobutylmethylxanthine, $10 \mu \mathrm{g} / \mathrm{ml}$ insulin and $1 \times 10^{-6} \mathrm{M}$ dexamethasone) for 3 days, followed by a 9-day incubation in maintenance media (growth medium plus $10 \mu \mathrm{g} / \mathrm{ml}$ insulin) with media replaced every other day. The cells were monitored daily using a microscope for the appearance of lipid droplets, which were confirmed by Oil-Red O staining as described previously ${ }^{15,40}$. Oil Red O solution was freshly made by diluting a stock solution ( $0.5 \mathrm{~g}$ of Oil Red O in $100 \mathrm{ml}$ of isopropanol) with water (6:4) followed by filtration.

\section{In vitro $B M M$ and $O C$ cultures}

Mouse BMMs and OCs were generated as described previously ${ }^{7,14,15,41}$. In brief, the bone marrow was flushed from femurs and tibiae of 2-3 month old WT and $\mathrm{APP}^{-1}$

mice with ice-cold $\alpha$-MEM and plated on $100 \mathrm{~mm}$ tissue culture plates in $\alpha$-MEM containing 10\% FBS and $10 \mathrm{ng} / \mathrm{ml}$ recombinant M-CSF (macrophage colonystimulating factor). Then cells were incubated at $37^{\circ} \mathrm{C}$ with $5 \% \quad \mathrm{CO}_{2}$ overnight. Non-adherent cells were harvested and subjected to Ficoll-Hypaque gradient centrifugation for purification of BMMs. For osteoclastogenesis, $1 \times 10^{5}$ BMMs were incubated with OC differentiation medium containing $10 \mathrm{ng} / \mathrm{ml}$ recombinant $\mathrm{M}-\mathrm{CSF}$ and $100 \mathrm{ng} / \mathrm{ml}$ recombinant RANKL (receptor activator of NFKB ligand). Mature OC (multi-nucleated, large spread cells) began to form at day 3-4 after RANKL treatment. The cells were then subjected to TRAP (tartrate-resistant acid phosphatase) staining to confirm OC identity.

\section{Transwell migration assay}

The migration ability of OBs isolated from long bone of $\mathrm{WT}$ and $\mathrm{APP}^{-1-}$ mice was examined in $6.5 \mathrm{~mm}$ transwell chambers with $8 \mu \mathrm{m}$ pores (Sigma). Briefly, $600 \mu \mathrm{l}$ DMEM supplemented with $10 \%$ FBS was added to the bottom chamber. Two hundred microliter resuspended cells $(1 \times$ $105 / \mathrm{ml}$ ) treated with compound in serum-free DMEM were added to the top chamber. After $8 \mathrm{~h}$, insert membranes were fixed with paraformaldehyde $4 \%(\mathrm{w} / \mathrm{v})$, nonmigrated cells removed with cotton swabs. Then the chamber was stained with crystal violet (Sigma) for $5 \mathrm{~min}$, membranes cut from the inserts and mounted using fluoroshield without DAPI. Migrated cells were counted in the entire insert membranes using a microscope (Leica Microsystems).

\section{Measurement of serum levels of osteocalcin and PYD}

Mouse blood was obtained by cardiac puncture. Samples were allowed to clot for at least $30 \mathrm{~min}$ and then centrifuged for $10 \mathrm{~min}$ at $3000 \mathrm{rpm}$. Serum was collected and frozen at $-20^{\circ} \mathrm{C}$ until use. Mouse serum levels of osteocalcin were measured by using mouse osteocalcin Elisa kit (QUIDEL Corporation). The serum levels of PYD were determined by using METRA Serum PYD EIA kit (QUIDEL Corporation). All the assays were carried out the instructions. All the ODs measured after reactions were converted to osteocalcin/PYD concentration using their standard curves. All the samples were measured in duplicate, and values were subjected to statistical analysis.

\section{Micro-computed tomography $(\mu \mathrm{CT})$}

Micro-architecture of the distal trabecular bone and midshaft cortical bone of the femur were measured by Scanco $\mu$ CT 40 (Scanco Medical AG, Brüttisellen, Switzerland) as described previously ${ }^{14,15,40-42}$. The 3-D reconstruction of the trabecular bone was performed using all the outlined slices. No cortical bone was included in this analysis. Data was obtained on bone volume (BV), total volume (TV), BV/TV, bone density, trabecular number, and connectivity. The scan of the cortical bone was performed at the midshaft of the femur and consisted of 25 slices (each slice was $12 \mu \mathrm{m}$ in thickness). Scans were reconstructed as for the trabecular scans and the region of interest was drawn on all 25 slices. There was no trabecular bone in these images at the midshaft. Cortical bone was thresholded at 329, and the 3-D reconstruction 
was performed on all 25 slices. Data was obtained on BV, $\mathrm{TV}, \mathrm{BV} / \mathrm{TV}$, bone density, and cortical thickness.

\section{Bone histomorphometric analysis}

Bone histomorphometric analyses were carried out as previously described ${ }^{14,15,40-42}$. In brief, mouse tibia and femurs were fixed overnight in $10 \%$ buffered formalin, decalcified in 14\% EDTA for 2 weeks, embedded in paraffin, sectioned, and subjected for H\&E, TRAP and Goldner's Trichrome stain analyses.

\section{Bone dynamic histomorphometric analysis}

To obtain the bone formation rate, P16 mice were injected with the fluorochrome labels calcein green (10 $\mathrm{mg} / \mathrm{kg}$, Sigma-Aldrich) intraperitoneally, and followed by another injection 12 days later. Mice were sacrificed two days after the second injection (P30). Mouse tibia and femurs were fixed overnight in $70 \%$ ethanol, embedded in OCT, cut $20 \mu \mathrm{m}$ frozen sections. Images were obtained using a $25 \times$ objective (LSM510; Carl Zeiss).

\section{Western blot assay}

Cells were lysed with RIPA lysis buffer $(50 \mathrm{mM}$ Tris$\mathrm{HCl}(\mathrm{pH} 7.4), 150 \mathrm{mM} \mathrm{NaCl}, 0.5 \%$ sodium deoxycholate, $1 \%$ Triton X-100, $0.1 \%$ SDS) containing protease and phosphatase inhibitor cocktails (Pierce). Cell and brain tissue lysates were centrifuged at $14,000 \times g$ for $20 \mathrm{~min}$ at 4 ${ }^{\circ} \mathrm{C}$. Equivalent proteins as determined by BCA assay (Pierce Biotechnology) were resolved by SDS-PAGE and subjected to western blotting with appropriate primary antibodies. All acquired images were subjected to densitometric quantitation using NIH Image software.

\section{Mitochondrial fractions}

Mitochondrial fractions were obtained as previously described $^{43}$. Briefly, BMSCs and OBs (D14, differentiation from BMSCs) from WT and $\mathrm{APP}^{-1-}$ mice were suspended in $0.5 \mathrm{mM}$ PMSF, $\mathrm{pH}$ 7.6) and homogenized by passing through a 27 -gauge needle 8 times. After incubation on ice for $15 \mathrm{~min}$, unbroken cells and nuclei were removed by centrifugation at $740 \mathrm{~g}$ for $10 \mathrm{~min}$. The supernatant was centrifuged at $9000 \times g$ for $10 \mathrm{~min}$ to obtain the cytosolic fraction (supernatant). The pellet was subjected to two additional rounds of homogenization and differential centrifugation. The mitochondrial fraction was pelleted by the final centrifugation at $14,000 \times g$ for $20 \mathrm{~min}$. Protein concentrations were measured by BCA kit from Pierce.

\section{Immunofluorescence staining and confocal imaging analyses}

Cells plated on coverslips in 12-well plates and fixed with $4 \%$ paraformaldehyde for $15 \mathrm{~min}$, permeabilized with $0.1 \% \mathrm{v} / \mathrm{v}$ Triton X-100/1xPBS $(10 \mathrm{~min})$, and blocked with $10 \%$ normal goat serum prior to incubation with primary antibodies overnight. Rabbit anti-ki67 (Millipore, 1:200), rabbit anti-Tom20 (Thermofisher, 1:1000), mouse anti-cytochrome $\mathrm{C}$ (BD, 1:500), rabbit anti-APP (Cell sig, 1:200), mouse anti-P62 (abcam, 1:500) and mouse anti-Tom20 (Santa Cruz, 1:100). Cells were then labeled with appropriate secondary antibody raised in goat (Invitrogen). Images were taken with the LSM800 (Zeiss) confocal microscope by using the $40 \times /$ 0.5 EC Plan-Neofluar objective or the $63 \times / 1.4$ Oil PlanApochromat objective. Excitation and acquisition parameters were constrained across all paired comparisons. For fluorescent quantification, morphometric measurements of images were performed using ImagePro Plus software (MediaCybernetics). Mitochondrial morphology quantification was conducted by the MitoMorphology Macro ${ }^{44}$ through ImageJ software (National Institutes of Health) as previous described ${ }^{43}$. In brief, images of 30 cells from random view field of each group were first processed with a median filter to obtain isolated and equalized fluorescence, then individual mitochondria were analyzed for the lengths of major axes.

\section{Mitochondrial functional assay}

Mitochondrial function was evaluated by oxygen consumption rate (OCR). It was measured in an X96 Extracellular Flux Analyzer with XF Cell Mito Stress Test Kit (Seahorse Biosciences) at $37^{\circ} \mathrm{C}$. BMSCs and OBs were plated on XF96 cell culture plate at $10 \mathrm{~K}$ per well and cultured for overnight with 80ul culture medium/well. Four wells without cells seeding from each plate were set as temperature and background control. For measurement, cells were gently rinsed with 100ul/well assay medium (XF Base medium (Seahorse Biosciences) with $2 \mathrm{mM}$ Glutamine and $10 \mathrm{mM}$ glucose), then put into $175 \mathrm{ul} /$ well assay medium and assayed. Three baseline OCR were calculated, followed by sequential injection of the ATP synthase inhibitor oligomycin, the mitochondrial uncoupler Carbonyl cyanide-4- (trifluoromethoxy) phenylhydrazone (FCCP) and the Complex I inhibitor rotenone. Two minutes OCR measurement were made at $3 \mathrm{~min}$ intervals with mixing and each condition was measured in each well.

\section{Discrimination of glycolytic versus OXPHOS ATP generation}

To mimic OXPHOS suppression, oligomycin, a potent inhibitor of the mitochondrial ATP synthase, was used. To inhibit ATP production by the glycolytic pathway, 2deoxyglucose (2DG) (the nonmetabolizable analog of glucose) was used. ATP level of BMSCs and OBs from $\mathrm{WT}$ and $\mathrm{APP}^{-/-}$mice without any treatment or treated 
with $25 \mathrm{mM}$ 2-DG or treated with $25 \mathrm{mM}$ 2-DG plus $5 \mu \mathrm{m}$ oligomycin for 45 min were measured with ATP Assay Kit (BioAssay Systems, Hayward, CA, USA) according to the manufacturer's instructions. Briefly, ATP released from cells immediately reacted with the substrate D-luciferin to produce light in the presence of luciferase and read luminescence on a luminometer within 1 min after adding reconstituted reagent.

\section{Determination of ROS}

The intracellular level of ROS is an important biomarker for oxidative stress and increased ROS level indicates increased oxidative stress generally. In this study, production of ROS was estimated with the fluorescent dye 2'7'-dichlorodihydrofluorescein diacetate $\left(\mathrm{H}_{2}\right.$ DCFDA; Thermofisher, Waltham, MA), a nonpolar compound that is converted by endogenous esterases to polar and membrane impermeable derivative $\mathrm{H}_{2} \mathrm{DCF}$. $\mathrm{H}_{2} \mathrm{DCF}$ is nonfluorescent but in the presence of intracellular ROS is oxidized to fluorescent $2^{\prime}, 7^{\prime}$-dichlorofluorescein (DCF). For measurement, BMSCs and OBs from WT and $\mathrm{APP}^{-1-}$ mice were washed once with PBS and loaded with $20 \mu \mathrm{M} \mathrm{H} \mathrm{H}_{2}$ DCFDA according to the manufacturer's instructions. After cells were incubated at $37^{\circ} \mathrm{C}$ for $30 \mathrm{~min}$ in the dark, the dye was removed, and cells were washed once with PBS and all of the samples were observed using confocal microscope immediately.

For measurement of mitochondrial $\mathrm{O}_{2}{ }^{-}$production, BMSCs and OBs cultured from 2-M WT and $\mathrm{APP}^{-1-}$ mice were loaded with $5 \mu \mathrm{M}$ MitoSOX (Invitrogen) in PBS containing $1 \mathrm{~g} / \mathrm{l}$ glucose for $10 \mathrm{~min}$ at $37^{\circ} \mathrm{C}$ incubator, washed with PBS for two times, and the fluorescence emission at $595 \mathrm{~nm}$ under $510 \mathrm{~nm}$ excitation was recorded using a microplate reader as previously described $^{45}$.

\section{Cell death assay}

The caspase family plays a key role in the molecular mechanisms of inducing cell death and is ultimately involved in apoptosis, among which caspase 3, also called "death protease", makes cell apoptosis inevitably once activated $^{46}$. So we did immunofluorescence staining of cleaved-caspase 3(rabbit, CST, \#9664S) to observe positive cells' percentage in both BMSCs and OBs from WT and $\mathrm{APP}^{-1-}$ mice.

\section{Transfestion of OBs with Mito-RFP and Sec61 $\beta$-GFP}

WT and $\mathrm{APP}^{-1-}$ OBs were and were plated at $1 \times 10^{4} /$ well onto 12-wells coverslips and grown in DMEM containing $10 \%$ (vol/vol) FBS, and 50 units $/ \mathrm{ml}$ penicillin and streptomycin plus $10 \mathrm{mM} \beta$-glycerophosphate and $50 \mu \mathrm{M}$ L-ascorbic acid-2- phosphate. At D10, cells were transfected with Mito-RFP and Sec61 $\beta$-GFP by Lipofectamine 3000 (Invitrogen), $72 \mathrm{~h}$ after transfection, cells were fixed and imaged by LSM800 (Zeiss) confocal microscope with the $63 \times / 1.4$ Oil Plan-Apochromat objective.

\section{MC3T3 cell culture and generation of APP-KO MC3T3 cell line}

MC3T3-E1 cells were grown in DMEM containing 10\% (vol/vol) FBS, and 50 units/ml penicillin and streptomycin. To differentiate pre-osteobalstic MC3T3 cells to mature osteoblastic MC3T3 cells, cells were induced by the addition of $50 \mathrm{ng} / \mathrm{ml}$ ascorbic acid and $10 \mathrm{mM} \beta$ glycerophosphate to standard growth medium for 7 days. All MC3T3 Cells were plated at $1 \times 10^{4} /$ well onto 12 -wells coverslips the day before transfection with LC3-GFP/ Mito-RFP/ CAG-LC3-mCherry-GFP plasmids.

For generation of APP-KO MC3T3 cell line, PX330 vector was purchased from Addgene. The puromycin resistant gene was replaced by GFP. Guidance RNA CACCGTGTATGCTGCCCGTTGGCCG for App was inserted into Bbsl restriction site and verified by sequencing. The PX330 plasmid was then transfected to MC3T3 cells and GFP positive cells were sorted by fluorescenceassociated cell sorting(FACS) and cultured in 96-well plate with one cell per well. 4-5 days later, the cells were passed to 12-well plate, and the App-KO clones were identified by western blot and PCR-based genomic DNA sequencing.

\section{RNA isolation and real-time PCR}

Total RNAs were isolated from primary cultured BMSCs, OBs (D14 culture) by Trizol extraction (Invitrogen, Carlsbad, CA, USA). Quantitative PCR was performed with Quantitect SYBR Green PCR Kit (Qiagen) according to the manufacturers' instructions and a RealTime PCR System with analytical software (Opticon Monitor 3). The following primers were used: P62, 5'-AC ATACGCAGAACAGAGTTACGAAGG-3' and $5^{\prime}$-CA TTCCAGTCATCTTGTCCGTAGGC-3'; GAPDH primers (5'-AAGGTCATCCCAGAGCTGAA $-3^{\prime}$ and $5^{\prime}$-CT GCTTCACCACCTTCTTGA-3' were used for normalization.

\section{NAC treatments}

For in vitro treatment, D1-OB cultures (differentiated from BMSCs) were treated with NAC (Sigma Aldrich, 1 $\mathrm{mM}$, overnight) or veh. control, then changed to $\mathrm{OB}$ differentiation medium. At D14, mitochondrial function assay and ALP staining were carried out.

For in vivo treatment, WT and $\mathrm{APP}^{-1-}$ mice were divided into two groups separately: (1) one group of mice $(n=5$, male) received drinking water, and (2) another group of mice $(n=5$, male) received drinking water containing NAC $(2 \mathrm{mg} / \mathrm{kg} /$ day $)$ for a period of 3-M. NAC in water $(0.001 \%, \mathrm{pH}$ adjusted to 7.2 by $\mathrm{NaOH})$ was prepared. Water was changed every other day for 3-M. 
Approximately $4-5 \mathrm{ml}$ of water was consumed by each mouse per day, which gives a cumulative dose $\sim 2 \mathrm{mg} / \mathrm{kg}$ per mouse per day. This NAC dose was chosen based on previous publications ${ }^{15,47}$. The NAC treatment was started at age of 1-2 $\mathrm{M}$ old. After 3-M of the treatment, mice were euthanized, and their femur bone and sera samples were collected for histomorphometric and $\mu \mathrm{CT}$ analyses, and for ELISA analysis of PYD and osteocalcin levels, respectively.

\section{Statistical analysis}

All assays were repeated in three independent experiments. All data were presented as mean \pm SEM. Significance was determined by Student's $t$-test for pairwise comparison and one-way ANOVA with Bonferroni posttest for multiple comparisons using GraphPad Prism 6.0 software (Graphpad Software, Inc., La Jolla, CA, USA). The significance level was set at $p<0.05$.

\section{Acknowledgements}

We thank members of the Xiong and Mei laboratories for helpful discussions. This study was supported in part by grants from National Institutes of Health (WCX and LM) and VA (WCX).

\section{Author details \\ ${ }^{1}$ Department of Neuroscience, Case Western Reserve University, Cleveland, $\mathrm{OH}$ 44106, USA. ${ }^{2}$ Department of Neuroscience and Regenerative Medicine, Medical College of Georgia, Augusta University, Augusta, GA 30912, USA. ${ }^{3}$ Charlie Norwood VA Medical Center, Augusta, GA 30912, USA. ${ }^{4}$ Louis Stokes Cleveland VAMC, Cleveland, $\mathrm{OH} 44106$, USA. ${ }^{5}$ The Center for Biomedical Research, Tongji Hospital, Tongji Medical College, Huazhong University of Science and Technology, 430030 Wuhan, China. ${ }^{6}$ Department of Rheumatology, Union Hospital, Tongji Medical College, Huazhong University of Science and Technology, 430022 Wuhan, China}

\section{Conflict of interest}

The authors declare that they have no conflict of interest.

\section{Publisher's note}

Springer Nature remains neutral with regard to jurisdictional claims in published maps and institutional affiliations.

Supplementary Information accompanies this paper at (https://doi.org/ 10.1038/s41419-018-1123-7).

Received: 5 January 2018 Revised: 12 September 2018 Accepted: 3 October 2018

Published online: 22 October 2018

\section{References}

1. Masters, C. L. et al. Alzheimer's disease. Nat. Rev. Dis. Prim. 1, 15056 (2015).

2. Zhou, R., Deng, J., Zhang, M., Zhou, H. D. \& Wang, Y. J. Association between bone mineral density and the risk of Alzheimer's disease. J. Alzheimers Dis. 24, 101-108 (2011).

3. Tysiewicz-Dudek, M., Pietraszkiewicz, F. \& Drozdzowska, B. Alzheimer's disease and osteoporosis: common risk factors or one condition predisposing to the other? Ortop. Traumatol. Rehabil. 10, 315-323 (2008).

4. Luckhaus, C. et al. Blood biomarkers of osteoporosis in mild cognitive impairment and Alzheimer's disease. J. Neural Transm. 116, 905-911 (2009).

5. Guerreiro, R., Bras, J. \& Hardy, J. SnapShot: genetics of Alzheimer's disease. Cell 155, 968-e1 (2013).
6. Small, S. A. et al. Model-guided microarray implicates the retromer complex in Alzheimer's disease. Ann. Neurol. 58, 909-919 (2005).

7. Xia, W. F. et al. Vps35 loss promotes hyperresorptive osteoclastogenesis and osteoporosis via sustained RANKL signaling. J. Cell. Biol. 200, 821-837 (2013).

8. Muller, U. C., Deller, T. \& Korte, M. Not just amyloid: physiological functions of the amyloid precursor protein family. Nat. Rev. Neurosci. 18, 281-298 (2017).

9. Esler, W. P. \& Wolfe, M. S. A portrait of Alzheimer secretases - new features and familiar faces. Science 293, 1449-1454 (2001).

10. Yan, R. et al. Membrane-anchored aspartyl protease with Alzheimer's disease beta-secretase activity. Nature 402, 533-537 (1999).

11. Bagyinszky, E., Youn, Y. C., An, S. S. \& Kim, S. Mutations, associated with earlyonset Alzheimer's disease, discovered in Asian countries. Clin. Interv. Aging 11, 1467-1488 (2016).

12. Hardy, J. Has the amyloid cascade hypothesis for Alzheimer's disease been proved? Curr. Alzheimer Res. 3, 71-73 (2006)

13. Hardy, J. Alzheimer's disease: the amyloid cascade hypothesis: an update and reappraisal. J. Alzheimers Dis. 9, 151-153 (2006).

14. Cui, $\mathrm{S}$. et al. APPswe/Abeta regulation of osteoclast activation and RAGE expression in an age-dependent manner. J. Bone Miner. Res. 26, 1084-1098 (2011).

15. Xia, W. F. et al. Swedish mutant APP suppresses osteoblast differentiation and causes osteoporotic deficit, which are ameliorated by $\mathrm{N}$-acetyl-L-cysteine. J. Bone Miner. Res. 28, 2122-2135 (2013).

16. Zheng, $H$. et al. beta-Amyloid precursor protein-deficient mice show reactive gliosis and decreased locomotor activity. Cell 81, 525-531 (1995).

17. Sims, N. A. \& Martin, T. J. Coupling the activities of bone formation and resorption: a multitude of signals within the basic multicellular unit. Bone Rep. 3, 481 (2014).

18. Wheater, G., Elshahaly, M., Tuck, S. P., Datta, H. K. \& van Laar, J. M. The clinical utility of bone marker measurements in osteoporosis. J. Transl. Med. 11, 201 (2013).

19. Chen, Q. et al. Fate decision of mesenchymal stem cells: adipocytes or osteoblasts? Cell Death Differ. 23, 1128-1139 (2016).

20. Cai, J., Yang, J. \& Jones, D. P. Mitochondrial control of apoptosis: the role of cytochrome c. Biochim. Biophys. Acta 1366, 139-149 (1998).

21. Anandatheerthavarada, H. K., Biswas, G., Robin, M. A. \& Avadhani, N. G. Mitochondrial targeting and a novel transmembrane arrest of Alzheimer's amyloid precursor protein impairs mitochondrial function in neuronal cells. J. Cell. Biol. 161, 41-54 (2003)

22. Gao, J. et al. SIRT3/SOD2 maintains osteoblast differentiation and bone formation by regulating mitochondrial stress. Cell Death Differ. 25, 229-240 (2018).

23. Chen, Y. et al. Tumour suppressor SIRT3 deacetylates and activates manganese superoxide dismutase to scavenge ROS. EMBO Rep. 12, 534-541 (2011).

24. Qiu, X., Brown, K., Hirschey, M. D., Verdin, E. \& Chen, D. Calorie restriction reduces oxidative stress by SIRT3-mediated SOD2 activation. Cell. Metab. 12, 662-667 (2010)

25. Chan, D. C. Dissecting mitochondrial fusion. Dev. Cell 11, 592-594 (2006).

26. lezaki, T. et al. The transcriptional modulator interferon-related developmental regulator 1 in osteoblasts suppresses bone formation and promotes bone resorption. J. Bone Miner. Res. 31, 573-584 (2016).

27. Filadi, R., Pendin, D. \& Pizzo, P. Mitofusin 2: from functions to disease. Cell Death Dis. 9, 330 (2018).

28. Rehklau, $\mathrm{K}$ et al. Cofilin1-dependent actin dynamics control DRP1-mediated mitochondrial fission. Cell Death Dis. 8, e3063 (2017).

29. Wu, S. B. \& Wei, Y. H. AMPK-mediated increase of glycolysis as an adaptive response to oxidative stress in human cells: implication of the cell survival in mitochondrial diseases. Biochim. Biophys. Acta 1822, 233-247 (2012).

30. Guntur A. R. et al. Osteoblast-like MC3T3-E1 cells prefer glycolysis for ATP production but adipocytelike 3T3-L1 cells prefer oxidative phosphorylation J. Bone Miner. Res. 6, 1052-1065 (2018).

31. Wang, K. \& Klionsky, D. J. Mitochondria removal by autophagy. Autophagy $\mathbf{7}$, 297-300 (2011).

32. de Brito, O. M. \& Scorrano, L. Mitofusin 2 tethers endoplasmic reticulum to mitochondria. Nature 456, 605-610 (2008).

33. de Brito, O. M. \& Scorrano, L. Mitofusin-2 regulates mitochondrial and endoplasmic reticulum morphology and tethering: the role of Ras. Mitochondrion $\mathbf{9}$ 222-226 (2009).

34. Sultana, R. \& Butterfield, D. A. Oxidative modification of brain proteins in Alzheimer's disease: perspective on future studies based on results of redox proteomics studies. J. Alzheimers Dis. 33, S243-S251 (2013). 
35. Pocernich, C. B. \& Butterfield, D. A. Elevation of glutathione as a therapeutic strategy in Alzheimer disease. Biochim. Biophys. Acta $\mathbf{1 8 2 2}$ 625-630 (2012).

36. Wang, X. et al. Amyloid-beta overproduction causes abnormal mitochondria dynamics via differential modulation of mitochondrial fission/fusion proteins. Proc. Natl Acad. Sci. USA 105, 19318-19323 (2008).

37. Vasconcellos, L. R. et al. Protein aggregation as a cellular response to oxidative stress induced by heme and iron. Proc. Natl Acad. Sci. USA 113, E7474-E7482 (2016).

38. Song, C. et al. Oxidative stress-mediated NFkappaB phosphorylation upregulates p62/SQSTM1 and promotes retinal pigmented epithelial cell survival through increased autophagy. PLOS ONE 12, e0171940 (2017).

39. Pocernich, C. B. \& Butterfield, D. A. Elevation of glutathione as a therapeutic strategy in Alzheimer disease. Biochim. Biophys. Acta 1822 625-630 (2012).

40. Zhang, W., Yang, N. \& Shi, X. M. Regulation of mesenchymal stem cell osteogenic differentiation by glucocorticoid-induced leucine zipper (GILZ). J. Biol. Chem. 283, 4723-4729 (2008).
41. Xiong, L. et al. Lrp4 in osteoblasts suppresses bone formation and promotes osteoclastogenesis and bone resorption. Proc. Natl Acad. Sci. USA 112 3487-3492 (2015)

42. Zhou, Z. et al. HMGB1 regulates RANKL-induced osteoclastogenesis in a manner dependent on RAGE. J. Bone Miner. Res. 23, 1084-1096 (2008).

43. Tang, F. L. et al. VPS35 deficiency or mutation causes dopaminergic neuronal loss by impairing mitochondrial fusion and function. Cell Rep. 12, 1631-1643 (2015).

44. Dagda, R. K. et al. Loss of PINK1 function promotes mitophagy through effects on oxidative stress and mitochondrial fission. J. Biol. Chem. 284, 13843-13855 (2009).

45. Suski, J. M. et al. Relation between mitochondrial membrane potential and ROS formation. Methods Mol. Biol. 810, 183-205 (2012).

46. Creagh, E. M. Caspase crosstalk: integration of apoptotic and innate immune signalling pathways. Trends Immunol. 35, 631-640 (2014).

47. Huang, Q. et al. Potential in vivo amelioration by N-acetyl-L-cysteine of oxidative stress in brain in human double mutant APP/PS-1 knock-in mice: toward therapeutic modulation of mild cognitive impairment. J. Neurosci. Res. 88, 2618-2629 (2010) 